\title{
Active Contour Models Based on Block Similarity for Multiple Objects Segmentation
}

\author{
Guoqi Liu (i) ${ }^{1,2}$ and Jinjin Wei ${ }^{3}$ \\ ${ }^{1}$ College of Computer and Information Engineering, Henan Normal University, Xinxiang 453007, China \\ ${ }^{2}$ Big Data Engineering Laboratory for Teaching Resources \& Assessment of Education Quality, Xinxiang 453007, Henan, China \\ ${ }^{3}$ College of Mathematics and Information Science, Henan Normal University, Xinxiang 453007, China
}

Correspondence should be addressed to Guoqi Liu; liuguoqi080408@163.com

Received 16 March 2019; Revised 21 September 2019; Accepted 14 October 2019; Published 6 November 2019

Academic Editor: Ludovico Minati

Copyright (C) 2019 Guoqi Liu and Jinjin Wei. This is an open access article distributed under the Creative Commons Attribution License, which permits unrestricted use, distribution, and reproduction in any medium, provided the original work is properly cited.

\begin{abstract}
For the model of active contours with group similarity (ACGS), a rank constraint for a group of evolving contours is defined to keep the shape similarity. ACGS obtains robust results in extracting a single object with missing or misleading features. However, with one initial contour, it could not extent to multiple objects segmentation because low-rank property will not hold in some image sequences. Besides, ACGS is affected by nontarget objects. In this paper, an active contour model based on block similarity of shapes is proposed to extend the ACGS model to realize multiple objects extraction. For a sequence of image with multiple objects, a model for multiple objects extraction is constructed by combining sparse decomposition and ACGS; second, a block low-rank constraint is proposed to constrain the similarity of these evolving contours in every block; finally, segmentation results are obtained through iterative evolutions. Experimental results show the proposed method could segment images with multiple targets, and it improves the robust segmentation performance of sequence of image when the features of multiobjects are missing or misleading.
\end{abstract}

\section{Introduction}

Object segmentation is an important research topic in many fields, such as computer vision and image recognition [1]. Compared with other methods [2,3], active contour models (ACMs) [4] have many advantages since the resultant contours are closed and quite regular, which are convenient for further applications $[5,6]$. The main principle of the $\mathrm{ACMs}$ is to define an energy functional and minimize the energy functional to obtain an optimal contour, and the converged result indicates the boundary of the target object to be extracted.

There are broadly two types of ACMs according to the representation of contour, i.e., parametric active contour model $[4,7]$ and geometric active contour model [8-12]. Geometric active contour model, which is also known as implicit active contour model, has been presented [8] based on level set method (LSM) $[8,10]$. LSM offers great flexibility for curve topology. Therefore, topological flexibility is a major advantage of geometric active contour model, which is desirable in detecting multiple objects [11].

Recently, geometric active contour models are widely researched and applied in medical image segmentation. For example, by integrating edge, region [12], or prior information into LSM, geometric active contour models could segment objects in various backgrounds, such as inhomogeneous intensity $[13,14]$ and object occlusion [15]. By combining edge and region information, distance regularized level set evolution (DRLSE) [16] and completely convex formulation of the Chan-Vese (C-V) [17] image segmentation model (CFCV) [18] have been proposed. Based on the distance regularized term, DRLSE could make the contour evolve robustly and segment multiple objects with proper initialization. With the convex relaxation method, the $\mathrm{CFCV}$ model is convex with respective to level set function, which is robust to initialization, and it greatly decreases the iterations 
and evolution time. In order to deal with inhomogeneous intensity, locally statistical active contour model (LSACM) [19] utilizes the local information to define a map function, which obtains robust segmentation performances.

In prior models based on LSM, shape prior $[20,21]$ is usually integrated into geometric active contour to segment or track objects. Shape prior has obtained robust results in segmenting objects with complex background [22-25]. However, the shape prior is usually learnt from a large set of annotated data, which is not always accessible in practice. Furthermore, the models with LSM usually have a large computational cost.

In order to avoid training of massive data and effectively deal with the affection caused by the interference information, an active contour with group similarity (ACGS) is proposed for single target extraction in a sequence of image [15]. It can be regarded as an unsupervised prior shape model. ACGS obtains robust results even in some object occlusion and noise. In ACGS, group similarity constraint is measured by low-rank, and low-rank property will not hold if the level set representation is used. Variational methods for image segmentation also have this issue [23-29]. Thus, parametric model is used to represent the contour in ACGS. In the parametric active contour model, contours are usually represented by landmarks. Generally, the parametric model is simple and fast. However, it could not segment multiple objects with one initial contour, since parametric representation cannot describe the topology change of contour. With multiple initial contours, parametric active contour could extract multiple objects. However, the disturbance of nontargets makes the contour farther away from the targets, and it is difficult to determine the locations and sizes of multiple initial contours during initialization.

In this paper, based on ACGS [15] and sparse decomposition method [30,31], ACGS is extended to realize multiple objects segmentation of image sequence with parametric active contour model. In the proposed method, the sparse decomposition method is integrated into ACGS to realize the multiple objects extraction of sequence of image and decrease the influence of nontarget objects. For the multiple evolving curves of a sequence of image, a block lowrank constraint is used to constrain the shape similarity of these evolving contours in every block.

The rest of this paper is organized as follows. Section 2 introduces some knowledge of ACMs. Problem analysis is stated in Section 3. The proposed model is described in Section 4. Section 5 demonstrates the merits of the proposed method by simulations. Finally, Section 6 concludes the paper with some discussions.

\section{Related Work}

2.1. Contour Evolution Framework Based on Parametric Active Contour Model. Let $I: \Omega \longrightarrow R$ denote a gray-level image, where $\Omega$ is the image domain and $R$ is a real number field. Let $C$ define a parametric curve in the $2-D$ plane $C=$ $\left[x_{1}, \ldots, x_{p}, y_{1}, \ldots, y_{p}\right]^{T} \in R^{2 p}$ and $p$ is the number of landmarks, $\left(x_{i}, y_{i}\right)$ is a landmark on the curve. In parametric ACM, a contour $C(s)=(x(s), y(s)), s \in[0,1]$ is represented by parametric form, and $s$ is a curve parameter. Assume $t$ is an artificial time, and $C(s, t)$ represents the evolving curve at time $t$. The initial curve $C(s, 0)$ imposed by a force function $F$ evolves to converge along its normal direction $\vec{N}$. The corresponding curve evolution equation is usually written as

$$
\left\{\begin{array}{l}
\frac{\partial C}{\partial t}=F(C, t) \vec{N}(C, t), \\
C(s, 0)=C_{0}(s) .
\end{array}\right.
$$

For parametric ACM, the Lagrange approach [32] is used to get the above evolution equation. By integrating the smooth constraint into parametric ACM, a curve is deformed to the object boundary by minimizing the following energy functional:

$$
E_{\text {snake }}=\int_{0}^{1} \frac{1}{2}\left(\alpha C_{s}^{2}(s)+\beta C_{s s}^{2}(s)\right)+E_{\text {ext }}(C(s)) \mathrm{d} s,
$$

where $C_{s}(s)$ and $C_{s s}(s)$ are the first and second derivatives of $C(s)$ with respect to parameter $s$ and $\alpha, \beta$ are the weighted constants. The external energy $E_{\text {ext }}$ is derived from the image $I$, which is usually computed as follows:

$$
E_{\text {ext }}(x, y)=-\left|\nabla\left[G_{\sigma}(x, y) * I(x, y)\right]\right|^{2},
$$

where $G_{\sigma}(x, y)$ is a two dimensional Gaussian function with a standard deviation $\sigma, G_{\sigma}(x, y)=\left(1 /\left(2 \pi \sigma^{2}\right)\right) e^{-\left(\left(x^{2}+y^{2}\right) / 2 \sigma^{2}\right)}$, $*$ is the convolution operation, and $\nabla$ is the gradient operator. Minimizing the above energy functional equation (2) with calculus of variations [32], an Euler equation is obtained as follows:

$$
\alpha C_{s s}(s)-\beta C_{\text {ssss }}(s)-\nabla E_{\text {ext }}(C(s))=0 .
$$

The first two terms are considered as an internal force, and $-\nabla E_{\text {ext }}$ is viewed as an external force imposing on the deforming curve. Many external force fields in parametric ACMs are proposed, such as gradient vector flow (GVF) vector field [33] and its improvements [34-36].

2.2. Active Contour with Group Similarity. According to the above section, the parametric ACM is usually used to extract a single object. When the object is partially occluded or corrupted by noise, the converged result may be undesired. In order to deal with this problem, ACGS [15] is proposed to robustly extract objects in a sequence of images. In ACGS, the shape conformability term for deforming contours is introduced. That is to say, the energy functional keeping the consistence of evolving contours is integrated into the classical energy functional of ACMs. In ACGS, the nuclear norm $\|X\|_{*}$, i.e., the sum of singular values of $X$, is introduced. The nuclear norm is a continuous and convex function, and some fast algorithms could be utilized. The energy functional $E_{\text {ACGS }}$ of the ACGS model is defined as follows:

$$
\min _{X} E_{\mathrm{ACGS}}(X)=\min _{X}\left\{G(X)+\lambda\|X\|_{*}\right\} .
$$

For the image sequence $I_{1}, \ldots, I_{m}$, the set $X=\left[C_{1}, C_{2}, \ldots, C_{m}\right]$ is constituted by a group of evolving 
contours and $m$ is the cardinality of $X$. $G$ is the energy functional of a general ACM, such as the parametric geodesic active contours (GAC) [9] and $\mathrm{C}-\mathrm{V}$ model [17], and $G(X)=\sum_{i=1}^{m} G\left(C_{i}\right)$. The energy functional $G$ of the parametric $\mathrm{C}-\mathrm{V}$ model in ACGS is defined as follows:

$$
\begin{aligned}
G_{\mathrm{cv}}(C)= & \int_{\Omega_{1}}\left(I(x)-c_{1}\right)^{2} \mathrm{~d} x+\int_{\Omega_{2}}\left(I(x)-c_{2}\right)^{2} \mathrm{~d} x \\
& +\gamma \text { length }(C),
\end{aligned}
$$

where $\Omega_{1}$ and $\Omega_{2}$ are the regions inside and outside the contour $C, c_{1}$ and $c_{2}$ are the mean intensities of $\Omega_{1}$ and $\Omega_{2}$ respectively, length $(C)$ represents the length of contour $C, \gamma$ is a parameter, and $G_{\mathrm{cv}}$ is usually less sensitive to initialization and has fewer parameters to tune, which makes contour evolve to object boundary.

Through a sequence of evolution $X_{1}, X_{2}, \ldots, X_{N}$, the converged contour $X_{N}$ is viewed as the final result. It is assumed that contour $C$ has $p$ points and the size of contour $C$ is $2 p$. Thus, the size of $X$ is $2 p \times m$. From equation (5), the shape conformability is kept and the robustness of evolution is improved since the constraint of nuclear norm $\|X\|_{*}$ is imposed. $\|X\|_{*}$ can keep the elements of $X$ similar. With the energy functional $G$ and the nuclear norm $\|X\|_{*}$, contours robustly evolve to the object boundary. Based on the gradient descent method, the evolution equation of $X$ in ACGS is iterated as follows:

$$
X_{l+1}=X_{l}-\Delta t w \nabla E_{\mathrm{ACGS}_{l}}
$$

where $X_{l}$ is the $l$-th iterative solution of $X, \Delta t$ is the time step, and $w$ is a parameter. From the above equation (7), $X_{l+1}$ is determined by $X_{l}$ and the gradient $\nabla E_{\mathrm{ACGS}}$.

\section{Analysis with ACGS}

ACGS is the kind of ACM which utilizes the CV model and combines with the constraint of the matrix's low-rank property. It mainly solves the segmentation problem of single similar target in a sequence of images when the target feature is missing or misleading. The realization process is expressed as follows: first, some discrete points are used to represent the evolving curves in each image. Then, the shape similarity of these curves is described by the nuclear norm. The similar object in the image group is segmented and restored by the relationship between the size of the matrix's rank and the similarity degree of target shape.

ACGS abandons the way of using level set function to evolve contours. Therefore, the ACGS model has the advantages of small computation and fast convergence. However, there are some problems to be considered:

(1) ACGS model introduces the low-rank constraint of the matrix. However, the low-rank property of the evolving contour with lever set method will not be valid. The ACGS model could not handle the change of topology, that is, the contour curve does not automatically split or merge. For multitarget sequence of image, ACGS cannot extract multitarget objects with one initial contour.
(2) Although multiple contours can be set to extract multitarget objects, it requires more information for initial contour position of each object. The contour evolution is always influenced by nontarget objects, and evolving contours may remain away from the target objects.

(3) ACGS is not robust to noise and nontargets, which greatly affects the smoothness of curve evolution.

\section{Active Contour Based on Block Similarity}

Aiming at the above problems of the ACGS model, in this paper, an active contour based on block similarity (ACBS) is proposed to extend the ACGS model. Firstly, based on ACGS, a model for multiple object extraction task is constructed, which is changed into some single object extraction tasks with sparse decomposition. Secondly, the block shape similarity for the multiple contours is integrated into the evolution equation. Finally, the converged contour is viewed as the final result.

4.1. The Model of ACBS. Assume that the number of the images in a sequence of image is $m$, i.e., $I=\left[I_{1}, \ldots, I_{m}\right]^{T}$. The number of objects of interest to be extracted from each image is $n$, i.e., $O=\left[O_{1}, \ldots, O_{n}\right]^{T}$. The evolving contours can be expressed as $B=\left[B_{1}, \ldots, B_{n}\right]^{T}, \quad B_{i}^{T}=\left[C_{i}^{1}\right.$, $\left.C_{i}^{2}, \ldots, C_{i}^{m}\right], i=1,2, \ldots, n$, and $C_{i}^{j}(i=1,2, \ldots, n ; j=$ $1,2, \ldots, m)$ is evolved in the $j$-th image to extract the $i$-th object. From the above definition,

$$
B=\left[\begin{array}{lll}
B_{1} & \cdots & B_{n}
\end{array}\right]^{T}=\left[\begin{array}{cccc}
C_{1}^{1} & C_{1}^{2} & \cdots & C_{1}^{m} \\
C_{2}^{1} & C_{2}^{2} & \cdots & C_{2}^{m} \\
\vdots & \vdots & \cdots & \vdots \\
C_{n}^{1} & C_{n}^{2} & \cdots & C_{n}^{m}
\end{array}\right],
$$

where $B_{i}(i=1,2, \ldots, n)$ is a block of contours, which includes $m$ contours. Every block $B_{i}(i=1,2, \ldots, n)$ evolves in the $m$ images to extract the corresponding objects which have similar shapes.

According to equation (8), the size of $B$ is $2 p * m * n$. With the above definitions, the proposed model is written as follows:

$$
\begin{aligned}
\min _{B} E_{\mathrm{ACBS}}(B) & =\min _{B}\left\{G(B)+\frac{\lambda}{2}\|B\|_{2, *}^{2}\right\}, \\
G(B) & =\sum_{i=1}^{n} G\left(B_{i}\right)=\sum_{i=1}^{n} \sum_{j=1}^{m} G\left(C_{i}^{j}\right),
\end{aligned}
$$

where $G$ is already defined in equation (5) and $\|B\|_{2, *}=$ $\left(\sum_{i=1}^{n}\left\|B_{i}\right\|_{*}^{2}\right)^{1 / 2}$ is a regularized term of evolving contours, which is used to ensure the shape similarity of these evolving contours in every block $B_{i}(i=1,2, \ldots, n)$.

With an alternating minimization method, the energy functional for the block $B_{i}(i=1,2, \ldots, n)$ is computed as follows: 


$$
\min _{B_{i}} E_{\mathrm{ACBS}}\left(B_{i}\right)=\min _{B_{i}}\left\{G\left(B_{i}\right)+\frac{\lambda}{2}\left\|B_{i}\right\|_{*}^{2}\right\}, \quad i=1, \ldots, n .
$$

With gradient descent method for equation (10), the following evolution equation with initialization is given as follows:

$$
\begin{cases}\frac{\partial B_{i}}{\partial t}=\nabla\left(G\left(B_{i}\right)+\frac{\lambda}{2}\left\|B_{i}\right\|_{*}^{2}\right), & i=1, \ldots, n, \\ B_{i}^{t}=B_{i}^{0}, & t=0 .\end{cases}
$$

According the above equation, initial block $B_{i}^{0}(i=1,2, \ldots, n)$ is evolved to converge to the object $O_{i}$ :

$$
B^{0}=\left[\begin{array}{llll}
B_{1}^{0} & B_{2}^{0} & \cdots & B_{n}^{0}
\end{array}\right]^{T}=\left[\begin{array}{cccc}
C_{1}^{1,0} & C_{1}^{2,0} & \cdots & C_{1}^{m, 0} \\
C_{2}^{1,0} & C_{2}^{2,0} & \cdots & C_{2}^{m, 0} \\
\vdots & \vdots & \cdots & \vdots \\
C_{n}^{1,0} & C_{n}^{2,0} & \cdots & C_{n}^{m, 0}
\end{array}\right]
$$

where $C_{i}^{j, 0}(i=1,2, \ldots, n ; j=1,2, \ldots, m)$ is the initial contour, which evolves in the $j$-th image to extract the $i$-th object.

According to equation (11), the evolution for every block is the same as the ACGS. The proposed method extends ACGS to the ACBS model for multiobject extraction. However, the difficulty lies in the initialization of every block. For a block, as shown in every column of matric $B^{0}$ in equation (12), there are $n$ initial contours to be set in one image.

4.2. The Evolution with ACBS. From the above ACBS model, initial contours evolve to segment the object targets. The initialization for ACBS is difficult since $B^{0}$ has $m * n$ contours. It is necessary to set $n$ initial contours for each image. For parametric ACM, it is difficult to set initial contours because there are some equilibrium points between objects [36]. By setting one initial contour, multiple objects extraction can be changed into multiple tasks of a single object extraction with sparse representation and decomposition method.

4.2.1. Sparse Decomposition. Contour cannot be split and merged automatically in the parametric ACM, thus the problem of multiobject segmentation cannot be divided. In the literature [37-39], the concept of sparse representation is introduced. Based on a dictionary [38] and sparse decomposition of signals, multiple object segmentation is obtained. The basic idea is the decomposition of signals on the overcomplete dictionary to get a simple representation. Sparse representation model is represented as follows:

$$
\begin{aligned}
Y & =D S=\sum_{i=1}^{\mathrm{NI}} D_{i} S_{i}, \\
D & =\left[D_{1}, \ldots, D_{\mathrm{NI}}\right], \\
S & =\left[S_{1}, \ldots, S_{\mathrm{NI}}\right] \sim \text { is } \sim \text { sparse }
\end{aligned}
$$

where $Y$ is a signal, $D$ is a set of basic functions or a dictionary, and $S$ is the coefficient matrix of the dictionary. Solving the above equation with some optimization methods [37] (such as greedy algorithm or convex relaxation method), the recovered signal $\widetilde{Y}$ represents as follows:

$$
\tilde{\mathbf{Y}}=\sum_{i=1}^{M} D_{i} S_{i}, \quad M \leq \mathrm{NI} .
$$

In order to explain the sparse decomposition method for object segmentation, the flow of sparse decomposition is described in Figure 1, and the corresponding example of the flow is shown in Figure 2. Based on the principle of sparse decomposition, the regional map (regional binary mask) of objects are firstly computed with a clustering operator. Clustering could group an image into some classes of similar characteristics. A clustering algorithm classifies the image into clusters, and the clusters are pairwise disjoint. The clustering result (cluster number is 3 in the classical fuzzy c-means clustering (FCM) [40]) is shown in the second image of the first row of Figure 2.

Secondly, a dictionary is obtained with a label operator for the clustering result. In label algorithm, several steps are used to label connected components. The matlab function "bwlabel" can be used to label each connected components. With a label operator, the connected region, in which pixels labeled 0 , is the background. The connected region of labeling 1 makes up a first object, the pixels labeled 2 are the second object, and so on. An example for labeling clustering result is shown in Figure 2. These labeled connected regions are viewed as the dictionary.

From the above two steps, the dictionary is obtained. That is to say that a global description function is decomposed into several local description functions:

$$
\begin{aligned}
f & =f_{L} S_{L}=f_{1}+f_{2}+\cdots+f_{\mathrm{NI}}, \\
f_{L} & =\left[f_{1}, f_{2}, \ldots, f_{\mathrm{NI}}\right], \\
\mathbf{S}_{L} & =[1,1, \ldots, 1],
\end{aligned}
$$

where $f_{1}, f_{2}, \ldots, f_{\mathrm{NI}}$ are the basic functions of the dictionary. Then, it is only necessary to obtain the basic functions of the target objects based on equation (15). Because some basic functions are redundant in the dictionary, the coefficients in the matrix $S_{L}$ are sparse (most of the coefficients are zero). Thus, the sparse coefficients are finally to be determined by solving equation (15) with the orthogonal matching pursuit (OMP) algorithm [37]. That is to say, equation (15) with sparsity constraint term of the coefficient matrix $\mathbf{S}_{L}$ is needed to solve based on the OMP algorithm. The main principle behind the OMP is as follows: within iteration, the coefficient $\mathbf{S}_{L}$ is computed based on the defined correlation function. The correlation function could be defined based on area or prior location information. With the OMP algorithm, two basic functions in the first two images of the second row of Figure 2 are used to extract the corresponding two objects. The segmentation result is shown in Figure 3. 


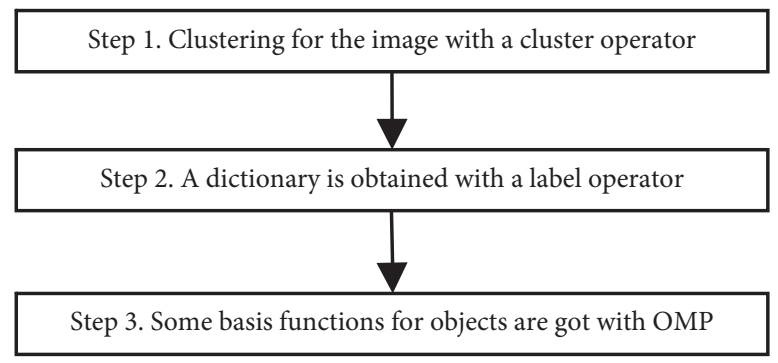

Figure 1: The flow of sparse decomposition.
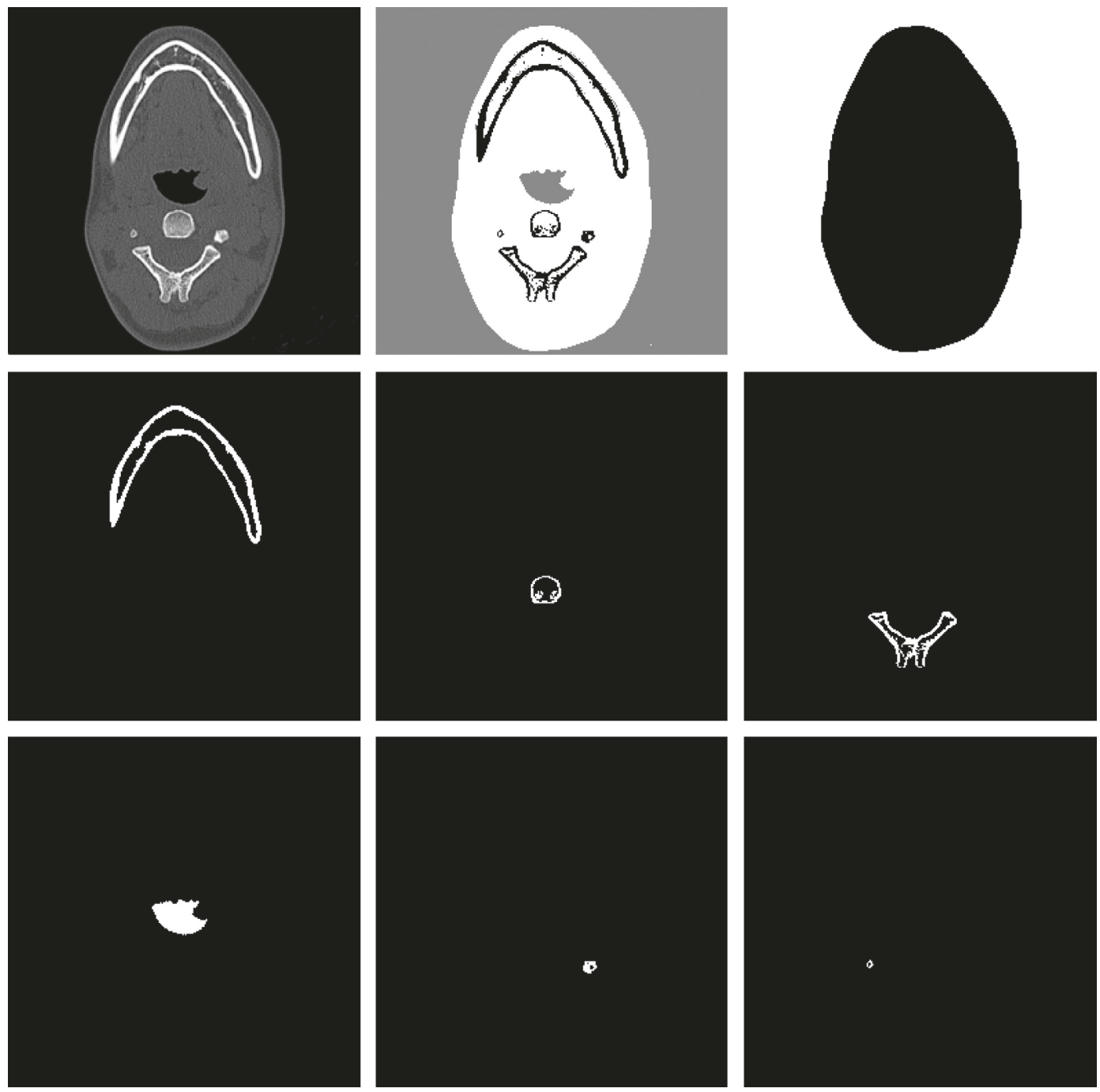

FIgURE 2: A medical image is used to explain the sparse decomposition method (the first image is a medical image, the second image shows the clustering result, and the rests are some basic functions with labeling for the clustering result.)

The basic functions of the two target objects are as follows:

$$
\tilde{f}=f_{1}+f_{2}
$$

that is, $M=2$ is set in equation (16). After the basic functions are obtained, new external forces $V_{i}(i=$ $1,2, \ldots, M)$ are generated according to [37]. After the external forces $V_{i}(i=1,2, \ldots, M)$ are computed, the evolution equation with the new external forces is computed as follows:

$$
\frac{\partial C}{\partial t}=\alpha C_{s s}(s)-\beta C_{s s s s}(s)+V_{i},
$$

with one initial contour $C$, the contour, respectively, evolves in external forces $V_{i}(i=1,2, \ldots, M)$ to converge to the corresponding objects boundaries $O_{i}(i=1,2, \ldots, M)$. Based 

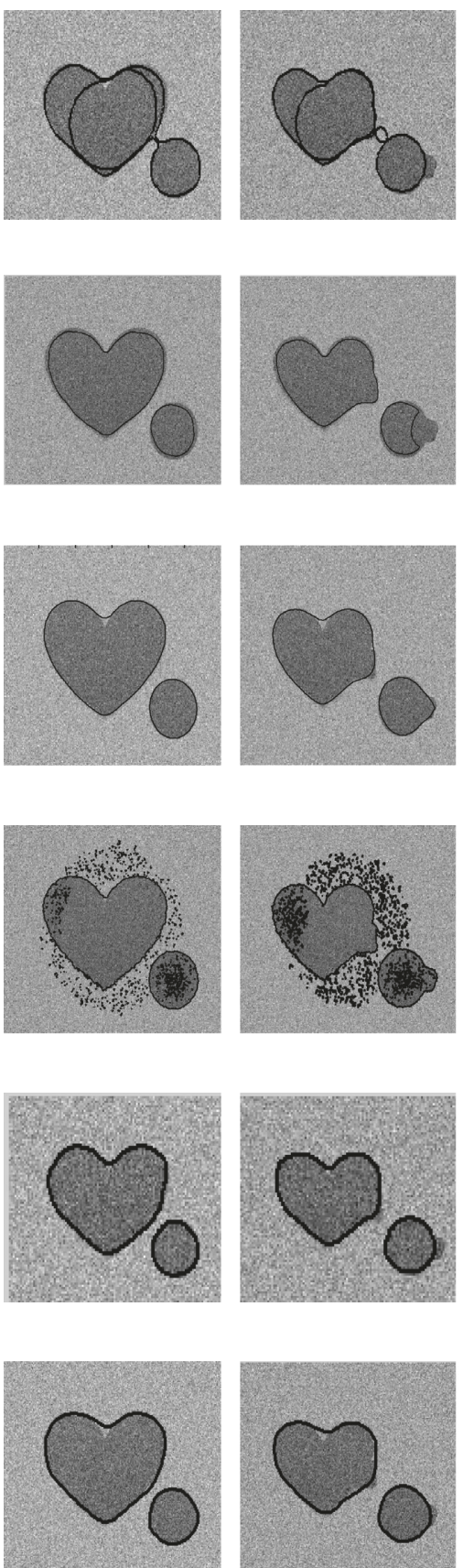
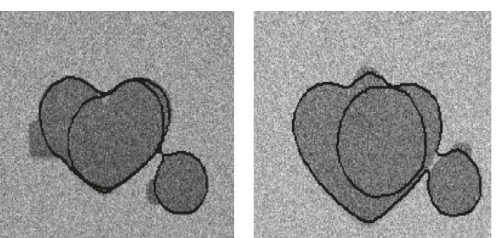

(a)
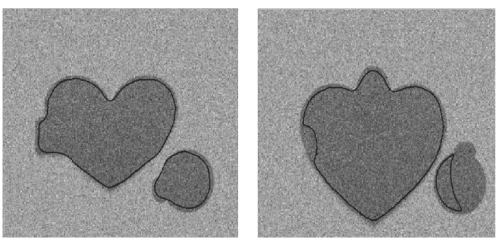

(b)
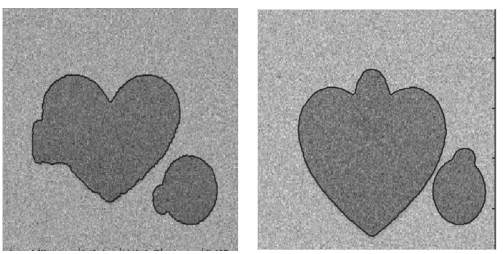

(c)
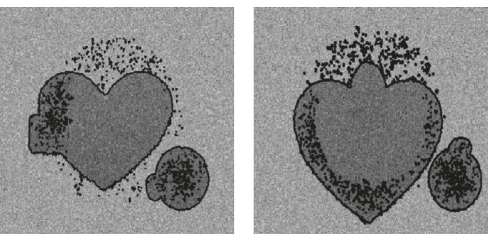

(d)
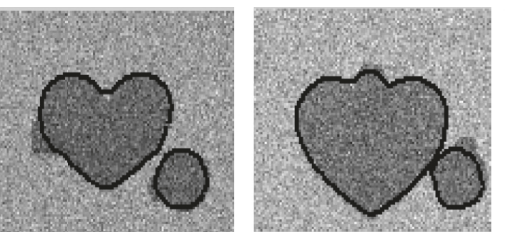

(e)

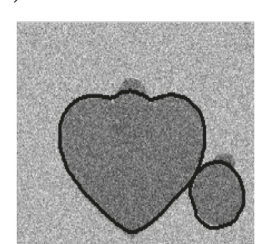

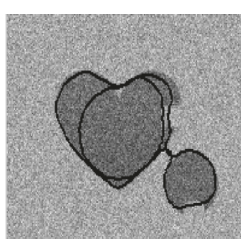
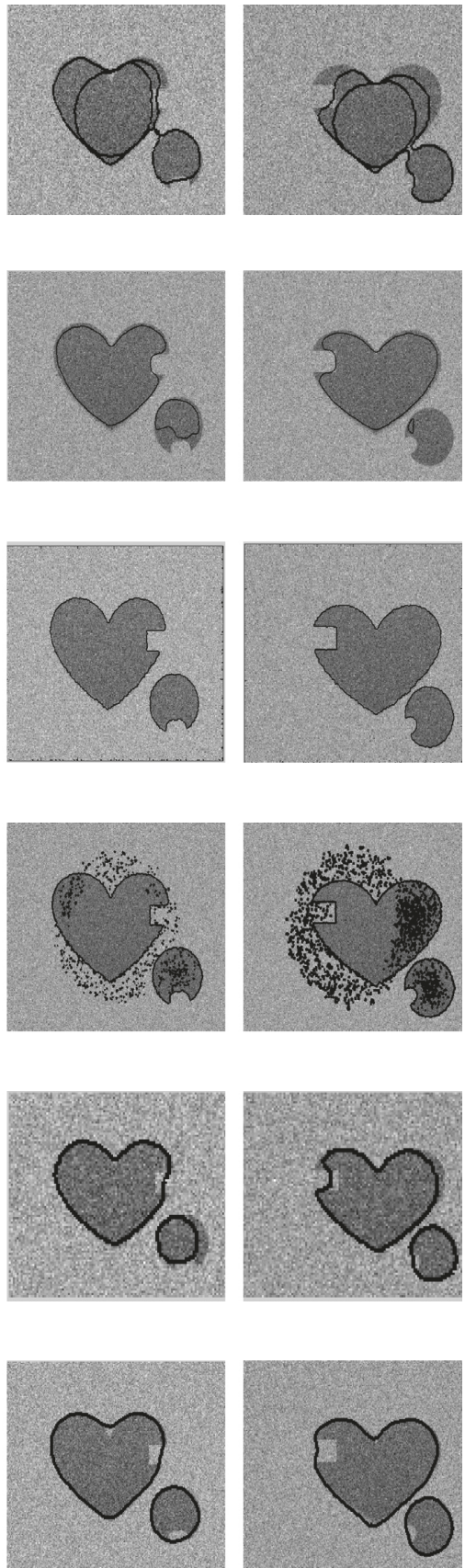

(f)

FIGURE 3: Segmentation results of synthetic image groups. The segmentation results of (a) the ACGS model, (b) the DRLSE model, (c) the CFCV model, (d) the LSACM model, (e) the ACGS model with initialization of basic functions, and (f) the proposed model.

on sparse decomposition, the basic function of each object is obtained and the influences from other objects are decreased, thus the contour of each target has been extracted.

4.2.2. Contour Evolution for a Sequence of Images. For a sequence of image with multiple objects, the model of ACBS is constructed by utilizing the sparse decomposition and block shape similarity constraint. As shown in Figure 4, an image sequence of image including six images is shown.
Every image in the sequence has two objects, and the objects appear loss or occlusion. The initial contour is also shown in each image. With the proposed method, the same initialization in every image is used. In the evolution, these contours are evolved and the nuclear norm constraint is imposed to maintain the shape similarity for each evolving contour.

According to equation (11), for the two objects extraction in Figure 4, the evolution equation of the two blocks is as follows: 

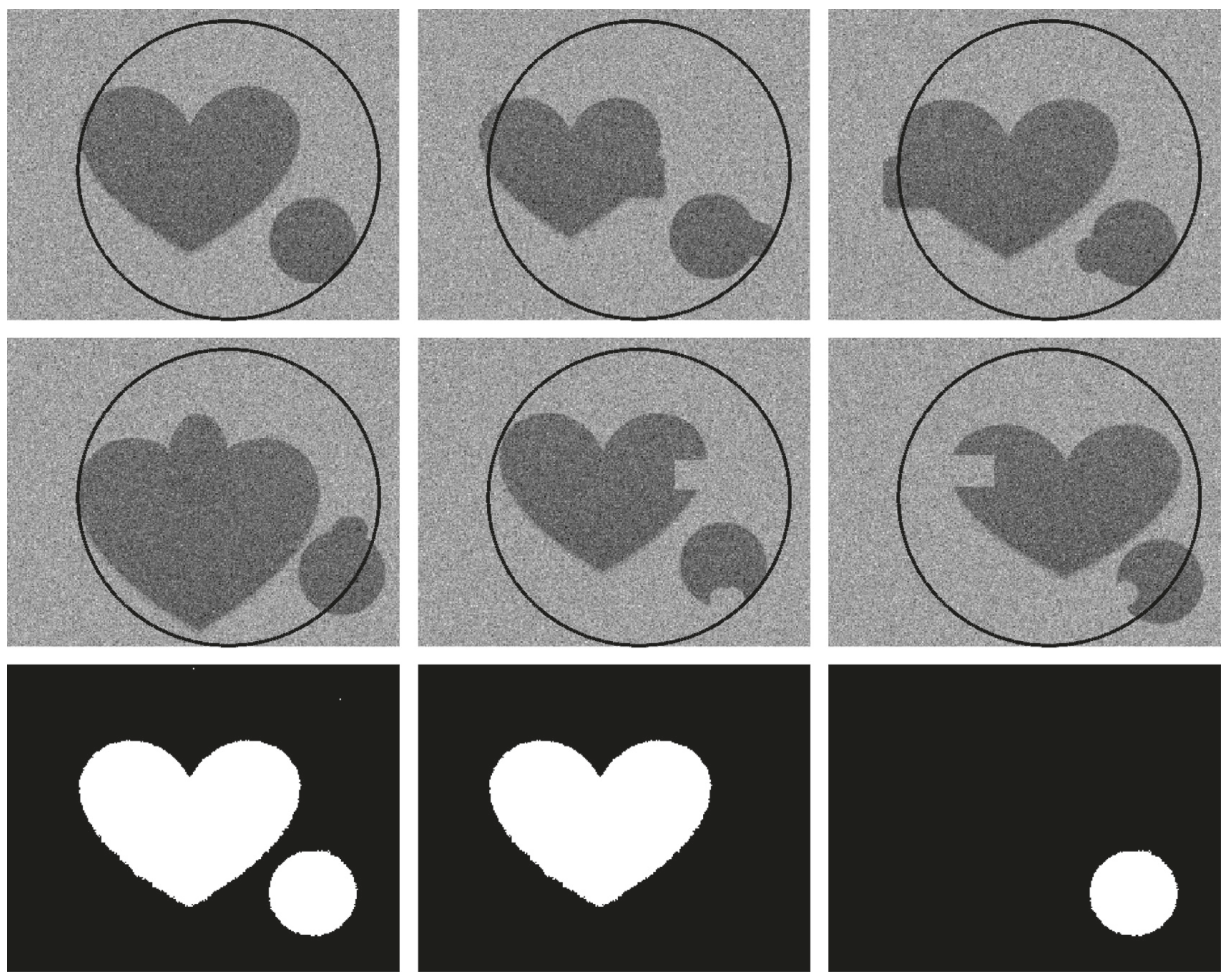

FIGURE 4: Every image in the sequence includes two objects, and the same initial contour is set in every original image. The first two rows are the original images with initialization, and the last row is the clustering result and two main basic functions for the first image.

$$
\begin{cases}\frac{\partial B_{i}}{\partial t}=\nabla\left(G\left(B_{i}\right)+\frac{\lambda}{2}\left\|B_{i}\right\|_{*}^{2}\right), & i=1,2, \\ B_{i}^{t}=B_{i}^{0}, & t=0,\end{cases}
$$

where $B_{i}^{0}(i=1,2)$ is evolved to converge to object $\mathrm{O}_{i}(i=1,2)$ :

$$
B^{0}=\left[B_{1}^{0}, B_{2}^{0}\right]^{T}=\left[\begin{array}{llll}
C_{1}^{1,0} & C_{1}^{2,0} & \cdots & C_{1}^{6,0} \\
C_{2}^{1,0} & C_{2}^{2,0} & \cdots & C_{2}^{6,0}
\end{array}\right]=\left[\begin{array}{llll}
C_{1} & C_{2} & \cdots & C_{6} \\
C_{1} & C_{2} & \cdots & C_{6}
\end{array}\right],
$$

where $C_{i}(i=1,2, \ldots, 6)$ represents the initial contour in the $i$-th image. For the target objects extraction, the clustering result and two basic functions are shown in the last row of Figure 4. Two blocks are used based on the sparse decomposition to converge to the corresponding objects. As shown in Figures 5 and 6, the evolution process for the corresponding object extraction is shown. The final column is the converged result with the proposed method. Compared with ACGS, one initial contour in every image is evolved to converge to multiple objects with ACBS.

In Figure 5, the contour evolution in the first block is shown to converge to the heart-shaped object. In Figure 6, the evolution in the second block is shown to extract the round-shaped object. In the evolution of every block, the shape similar constraint of the evolving contours is used to keep the shape conformability, thus it keeps the robust segmentation performance of sequence of image when the features of multiobjects are missing or misleading. The evolution process is shown in the first three columns of Figures 5 and 6 . With the proposed method, the converged results are shown in the last column of Figures 5 and 6 . Though the objects in the sequence of image suffer from noise and occlusion, the shapes of extracted objects in every block are consistent.

\section{Experiments and Analysis}

The main part of this section is to verify the segmentation performance of the proposed model and the advanced models through the simulation experiments in MATLAB R2016a software, and the contrast models include the ACGS model, the LSACM model, DRLSE model, and CFCV model. ACGS is the typical model of parametric active contour model, and LSACM is the typical one in geometric active contour model to deal with inhomogeneous intensity. Both DRLSE and CFCV models are classical segmentation methods, which utilize the edge and region information to extract multiple objects, and robustly evolve. Thus, these four models are tested and compared. The external force utilized in the proposed model and ACGS model is the parametric $\mathrm{C}-\mathrm{V}$ model. The parameters in each model are usually set to default values.

The tested image dataset used in this paper mainly consists of synthetic multitarget sequence of images and CT scanning sequence of images whose pixels are $300 \times 240$ and $512 \times 512$, respectively. The contour of dental lamina in the dental CT images is needed to be extracted. The initial contour is set to a circular curve and contains the target objects to be extracted. The size and position of the initial 

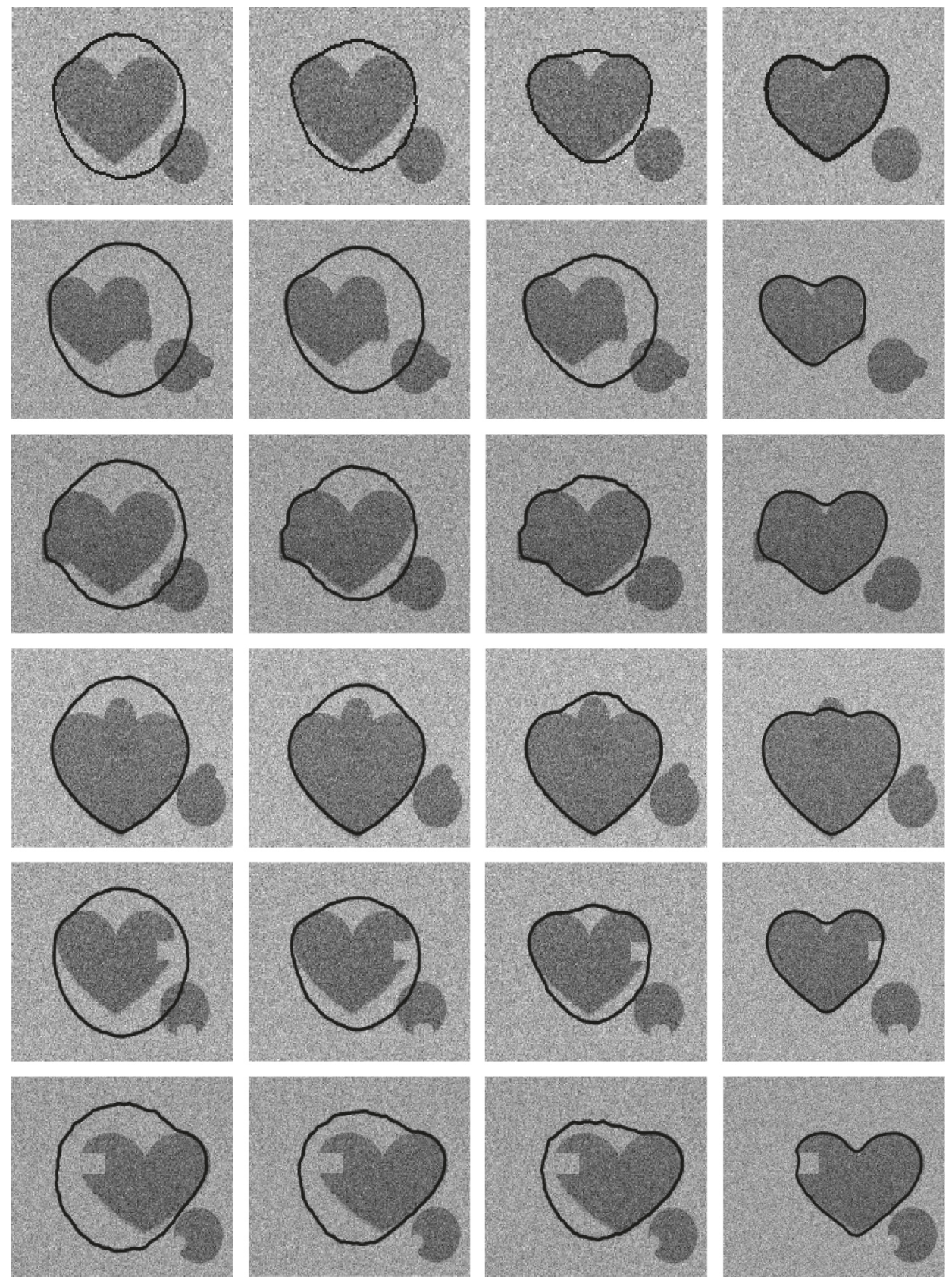

FIGURE 5: The process of converging to the heart-shaped object (with some feature missing or misleading) in a sequence of image (the last column is the final result).

contour of each sequence of image used for testing remain unchanged. Then, the endocardium segmentation of the left ventricle in cardiac cine MRI is tested. The dimension of the images is $192 \times 156$, and slice thickness is $8 \mathrm{~mm}$ and the spatial resolution is $1.82 \times 1.82 \mathrm{~mm}$ in-plane. Finally, the T1weighted images are tested, and the images can be downloaded on the line http://www.cma.mgh.harvard.edu/ibsr/. Six images from an image sequence of the same scan are tested. The dimension of the images is $256 \times 256$. The pixel resolutions on these images are $0.9375 \times 0.9375 \mathrm{~mm}$ in-plane by $3.1-\mathrm{mm}$ slice thickness.
In order to test the segmentation performance of a multitarget sequence image, a classical toy example is tested, which has been simulated in ACGS. Toy example is the synthetic images that contain a heart-shaped object. Some heart-shaped objects are to simulate missing or misleading situations. They have different sizes and positions in each image, and Gaussian noise is added to each image. On the basis of these images, we added a circular target in each image and simulated missing or misleading phenomena, respectively. They are also different in their position and size. Six synthetic images with multiple targets 

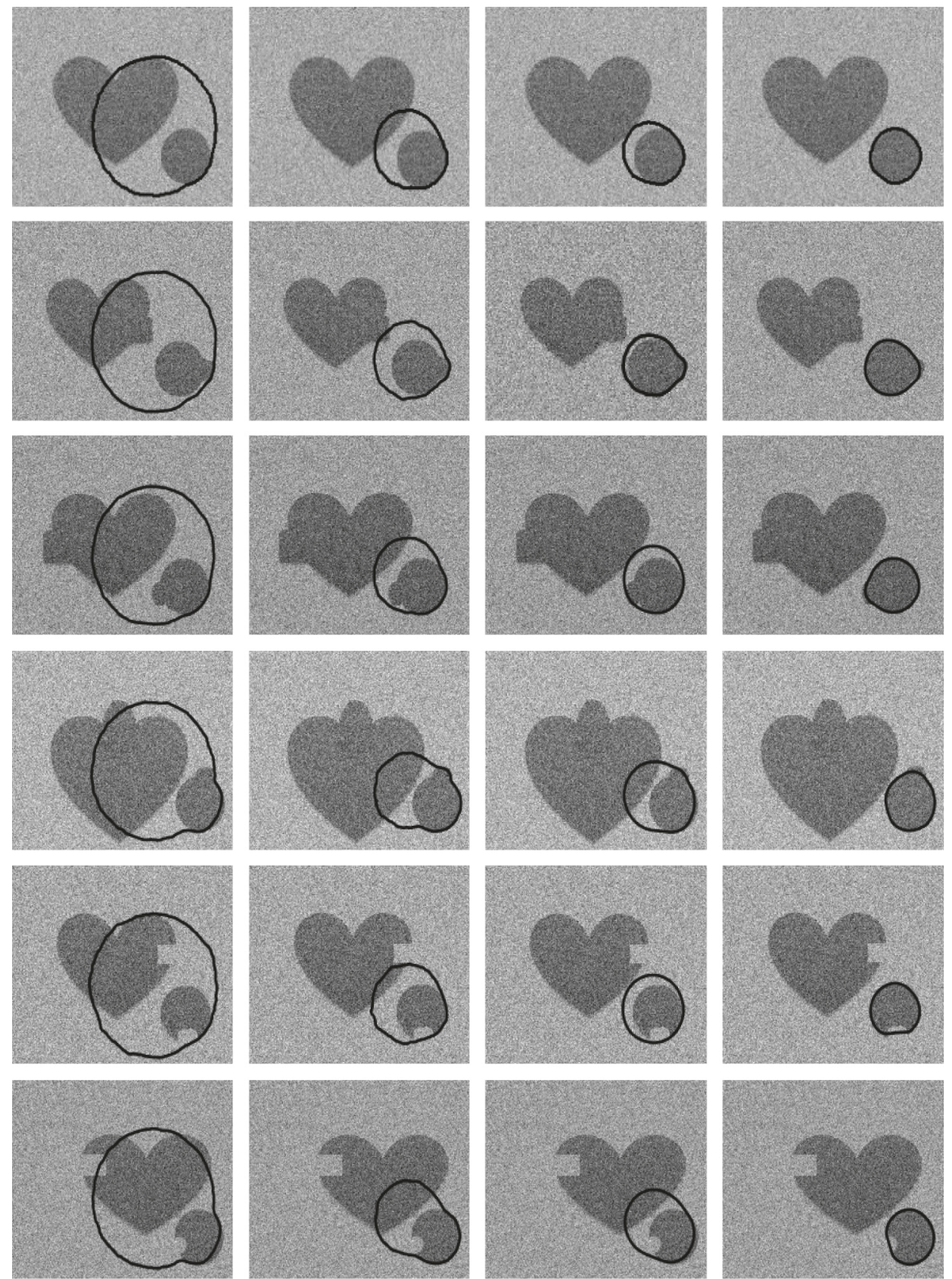

Figure 6: The process of converging to the round-shaped object (with some feature missing or misleading) in a sequence of image (the last column is the final result).

are selected to simulate. The segmentation results are shown in Figure 3.

Figure 3(a) is the segmentation result with the ACGS model. It can be seen that the ACGS model is not capable of achieving ideal segmentation results for multitarget images. On one hand, contour in the ACGS model could not automatically split or merge. Therefore, an initial contour cannot accurately extract multitarget objects. On the other hand, the images in the tested sequence of image have different shapes of objects, and the shapes of these whole objects are greatly different. Thus, group shape similarity constraint does not hold for multiple objects. As shown in the 4-th, 5-th, and 6-th columns in Figure 3(a), the evolving contours in these images are affected by the missing or misleading feature, and they cannot maintain the original shape. Furthermore, self-crossing problems always appear when parametric active contour suffers from noise. As shown in the first row of Figure 3, contour in the evolution appears self-crossing with ACGS.

The segmentation results with the DRLSE, CFCV, and LSACM models are, respectively, shown in Figures 3(b)3(d). The LSACM model uses the statistical information of images to build a level set energy functional, which is insensitive to initialization of level set. The CFCV model is a 
convex formulation of $\mathrm{C}-\mathrm{V}$ model, and it is also robust to initialization. DRLSE depends on initialization, and it should be set outside or inside the objects. However, group similarity is measured by low-rank constraint, and lowrank property will not hold if the level-set representation is used. Thus, the shape similarity constraint is not suitable for DRLSE, CFCV, and LSACM models. As can be seen from the Figures 3(b)-3(d), however, these models make the contour automatically split and merge and successfully extract multiobjects. However, the rank constraint of matrix could not be imposed in these models. That is, they cannot solve the segmentation problem of similar sequence of image with multitarget object feature missing or misleading. Furthermore, the LSACM model is not robust to noise. Thus, nontargets and noise points are extracted. As shown in Figure 3(d), some noises are extracted with the LSACM model. By adjusting the regularized parameters of $\mathrm{CFCV}$, the effect of noises could be decreased. Compared with LSACM, the CFCV is robust to noise, as shown in Figure 3(c).

Compared with the above models, the segmentation results with the proposed ACBS model are shown in Figure 3(f). It can be clearly seen that the model can effectively solve the segmentation problem of multitarget objects when the target features are missing or misleading, and the segmentation results are more consistent with the target object to be extracted. Compared with ACGS, the proposed model improves the robustness of evolution, and self-crossing in the evolution disappeared.

ACGS initialized with the basic functions is also tested, which is shown in the Figure 3(e). Compared with the original ACGS model, ACGS initialized with the basic functions has obtained better results. Because of the ideal initialization with basic functions, the self-crossing in the evolution decreases. However, for the multiple object segmentation, it needs multiple initial contours. Furthermore, ACGS is still affected by other objects and noise. Thus, the accuracy with ACGS is less than the one with the proposed method. Since the idea of sparse decomposition is introduced, the affection of noise and nontargets is greatly decreased, and the smoothness of evolving contours in the proposed model is enhanced. On the basis of this, the shape similarity constraint for every block is combined to maintain the shape consistency of the evolving contours. The quantitative assessment for tested methods is shown in Tables 1 and 2.

Then, the tested models are applied on the CT sequence of images. The simulation results of the sequence of images are listed and shown in Figure 7. CT images contain many objects and noises, and the contour of dental lamina is concave and it is difficult to realize the segmentation of the objects in the sequence of images.

For the ACGS model, the segmentation results (which are shown in Figures 7(a) and 7(e)) are not ideal. The ACGS model is affected by the nontarget objects, and the target objects are not extracted. With the initialization of basic functions of target objects, the segmentation is still greatly affected by nonobjects. As shown in Figure 7(e), the segmented boundaries are not ideal.
The LSACM model takes advantage of the statistical information of the image, and it does not rely on the edge information of the image. It extracts multitarget objects. However, it is sensitive to noise and easy to extract nontarget objects, and the model relies on specific probabilistic models, and the computation complexity is high. As shown in the second row of Figure 7, some noise and nontarget objects are also extracted. CFCV model extracts the objects from the background. However, some noises and nonobject are also extracted. The shape similarity is not maintained, which is shown in Figure 7(c). By setting initial contour outside the objects, the DRLSE model could extract the objects inside the initial contour. However, DRLSE is influenced by other objects and background. The converged contours are undesired. The segmentation results with the proposed model are shown in the last row of Figure 7. The proposed method successfully extracts multitarget from sequence of images even though some boundaries of objects are missing.

The tested methods are also used to apply the endocardium segmentation of the left ventricle. In Figure 8, an image is shown, and the corresponding clustering result and some basic functions are also shown. There are some weak boundaries near the endocardium. The segmented result with DRLSE is affected by other objects, and contour evolves to other objects, which is shown in Figure 9(b). For the LSACM and CFCV models, both of them extract the target objects, but some nonobjects are also extracted though the initialization is near the target objects, which are shown in the Figures 9(c) and 9(d). ACGS and the proposed method succeeded in extracting the endocardium, which are shown in Figures 9(a) and 9(e). The zoomed comparisons for ACGS and our method are shown in Figure 10. Compared with the proposed method, some areas are not extracted with ACGS.

Finally, the tested methods apply on the multiple images of a patient with a tumor in the same scan. The outline of brain and tumor boundary is to be extracted. The first row is the original image sequence. The shapes of the object (tumor) are less similar in the first three images. Inhomogeneous intensity appears inside the tumor, which is shown in the first image of Figure 11. The corresponding clustering result also shows some parts of the tutor are not detected because of inhomogeneous intensity.

With the default parameters, the result is shown in the second row of Figure 12. ACGS keeps the shape consistent, but boundary leakage appears. DRLSE extracts some contours of the tumor, but weak boundary leakage appears in the first three images. For the LSACM model, since the tumor is very small compared with other objects and background, the tumor is completely neglected, which is shown in the fourth row of Figure 12. For the CFCV model, as shown in the fifth row of Figure 12, affected by other objects, the extracted result is also not ideal.

The last two rows are the converged results with the proposed method by setting different regularized parameters. According to [15], the larger $\lambda$ makes the converged contours more similar to each other. When the shapes of target objects are less similar, the parameter can be set to small. The zoomed comparisons for the fourth image with the proposed method in Figure 12 are shown in Figure 13. 
TABLE 1: Comparisons of segmentation metric with tested models.

\begin{tabular}{|c|c|c|c|c|c|c|c|c|c|c|c|c|c|c|c|c|c|c|}
\hline & \multicolumn{3}{|c|}{1} & \multicolumn{3}{|c|}{2} & \multicolumn{3}{|c|}{3} & \multicolumn{3}{|c|}{4} & \multicolumn{3}{|c|}{5} & \multicolumn{3}{|c|}{6} \\
\hline & $\mathrm{P}$ & $\mathrm{R}$ & $\mathrm{F}$ & $\mathrm{P}$ & $\mathrm{R}$ & $\mathrm{F}$ & $\mathrm{P}$ & $\mathrm{R}$ & $\mathrm{F}$ & $\mathrm{P}$ & $\mathrm{R}$ & $\mathrm{F}$ & $\mathrm{P}$ & $\mathrm{R}$ & $\mathrm{F}$ & $\mathrm{P}$ & $\mathrm{R}$ & $\mathrm{F}$ \\
\hline ACGS & 86.01 & 96.33 & 90.88 & 87.30 & 98.11 & 92.39 & 92.10 & 87.39 & 89.69 & 82.31 & 92.60 & 87.15 & 81.82 & 97.98 & 89.17 & 90.29 & 98.76 & 94.34 \\
\hline LSACM & 83.59 & 98.57 & 97.66 & 84.55 & 97.72 & 90.66 & 84.56 & 94.17 & 89.11 & 78.14 & 93.18 & 85.00 & 90.46 & 80.99 & 69.19 & 82.20 & 97.84 & 89.34 \\
\hline DRLSE & 86.88 & 99.93 & 92.95 & 78.01 & 96.57 & 86.30 & 78.05 & 99.99 & 87.67 & 67.95 & 99.99 & 80.92 & 81.99 & 96.65 & 88.71 & 89.85 & 95.73 & 92.70 \\
\hline CFCV & 96.22 & 98.17 & 97.19 & 96.28 & 95.50 & 95.88 & 92.16 & 99.13 & 95.52 & 94.22 & 95.02 & 94.62 & 94.42 & 95.70 & 95.05 & 97.27 & 92.66 & 94.91 \\
\hline $\operatorname{ACGS}(\mathrm{B})$ & 93.53 & 99.20 & 96.28 & 93.08 & 94.29 & 95.14 & 85.73 & 99.06 & 91.91 & 85.28 & 99.74 & 91.95 & 93.51 & 98.19 & 95.79 & 93.89 & 97.98 & 95.89 \\
\hline Ours & 94.10 & 99.37 & 96.66 & 92.72 & 98.84 & 95.68 & 94.64 & 97.44 & 96.02 & 94.99 & 98.12 & 96.53 & 91.96 & 98.77 & 93.61 & 94.41 & 99.39 & 96.69 \\
\hline
\end{tabular}

TABle 2: Comparisons of the averaged F-Score with tested models.

\begin{tabular}{lcccrr}
\hline \multicolumn{4}{c}{ Averaged F-Score } \\
ACGS & LSACM & DRLSE & CFCV & ACGS(B) & Ours \\
\hline 90.6 & 86.83 & 88.21 & 95.53 & 94.49 & 95.87 \\
\hline
\end{tabular}
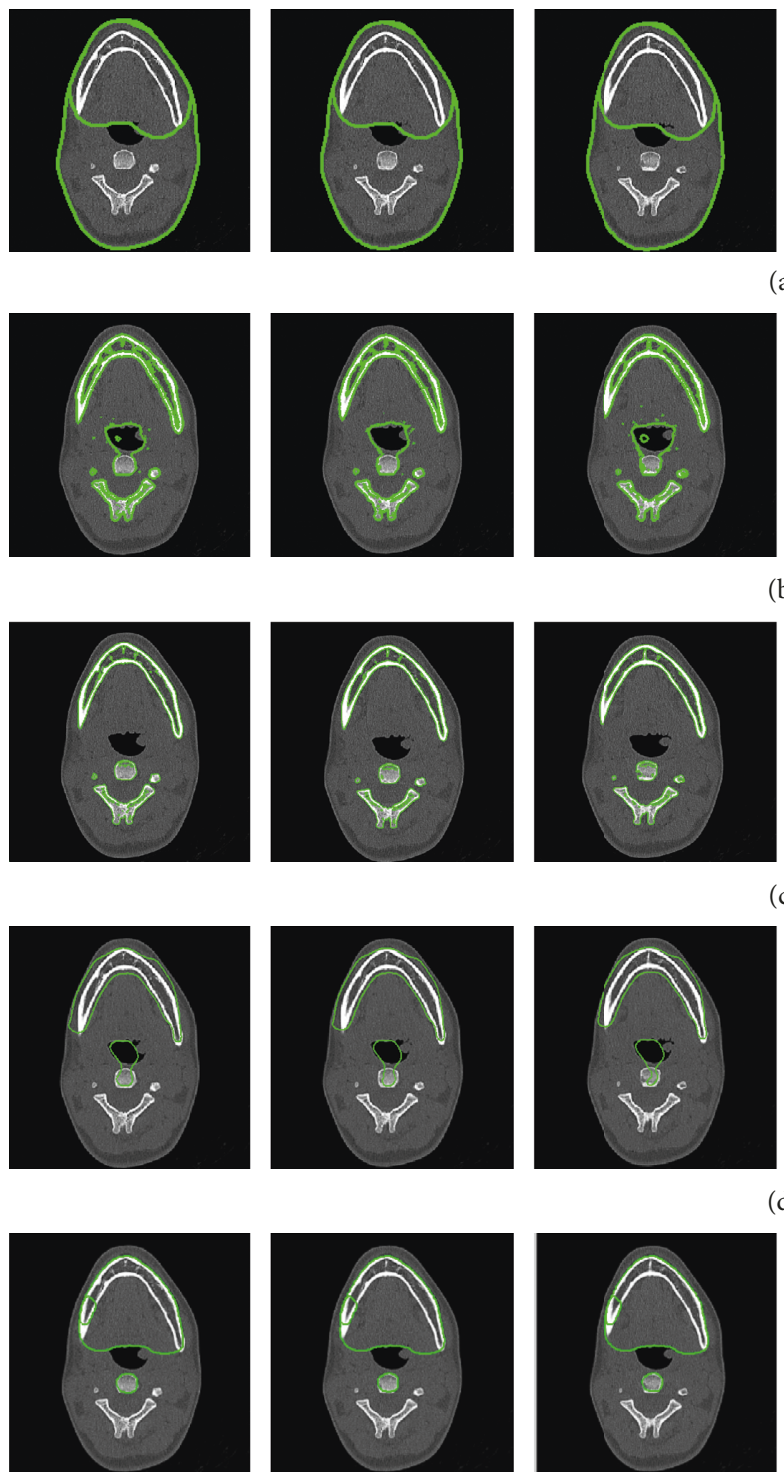

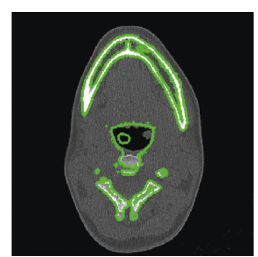

(b)

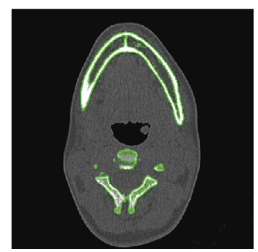

(c)

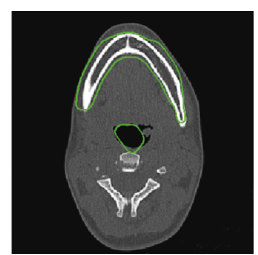

(d)
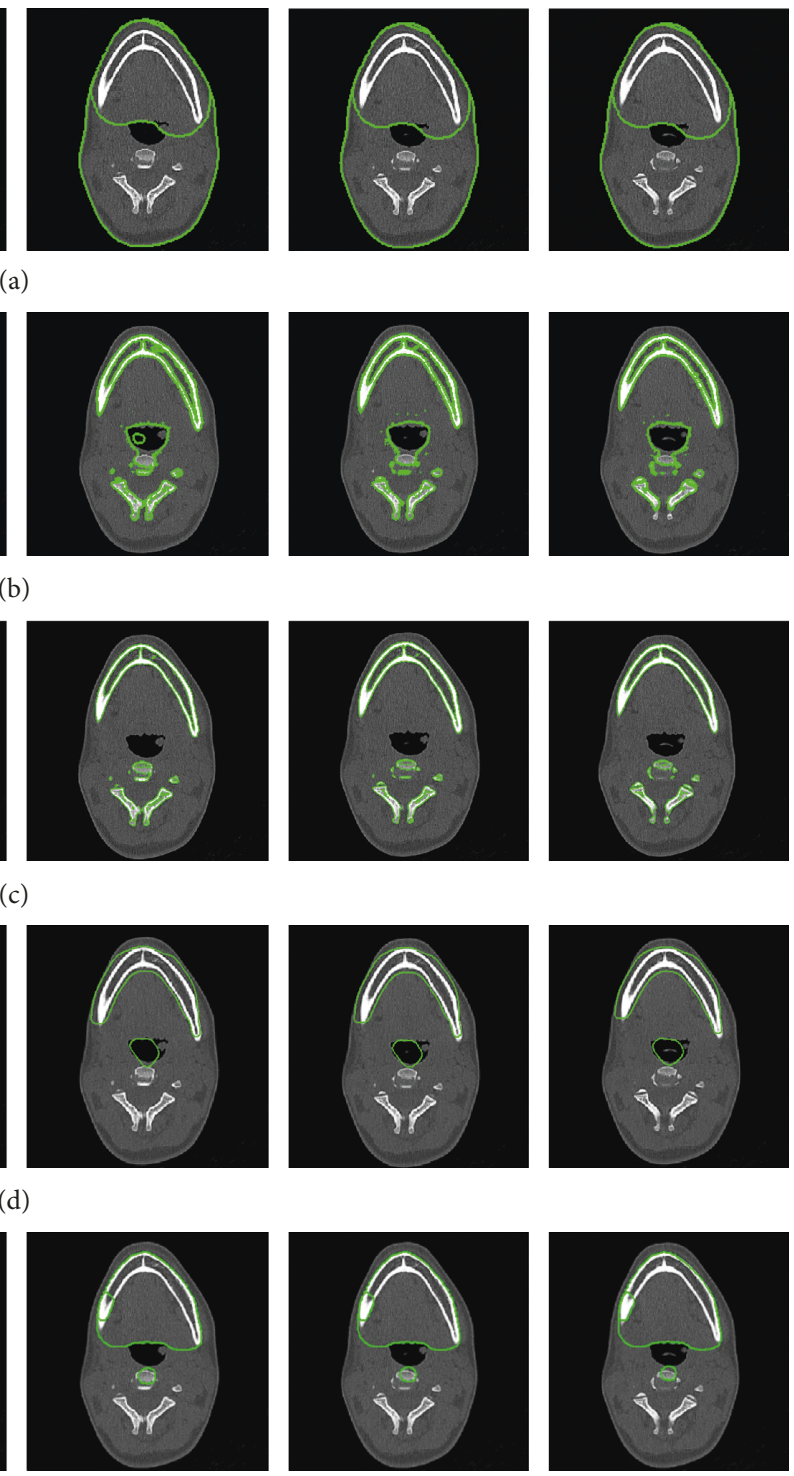

(e)

FIgURE 7: Continued. 

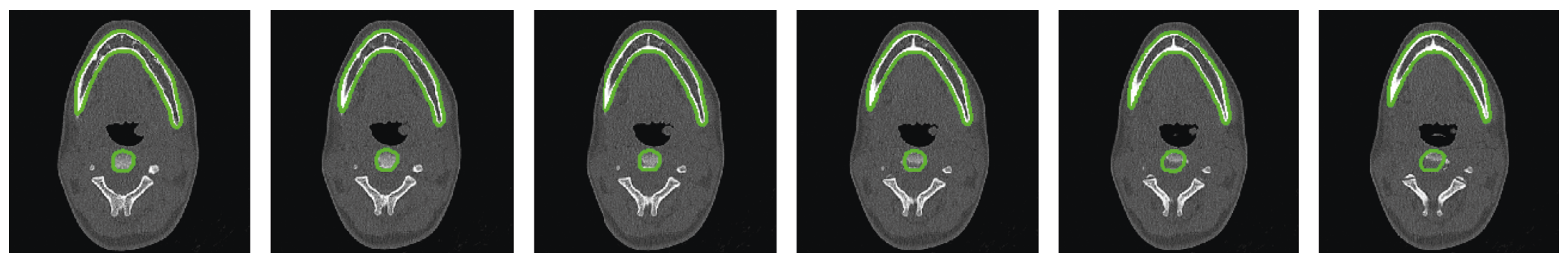

(f)

FIGURE 7: Segmentation results of the dental CT sequence of images. The segmentation results of (a) the ACGS model, (b) the LSACM model, (c) the CFCV model, (d) the DRLSE model, (e) the ACGS model with initialization of basic functions, and (f) the ACBS model.
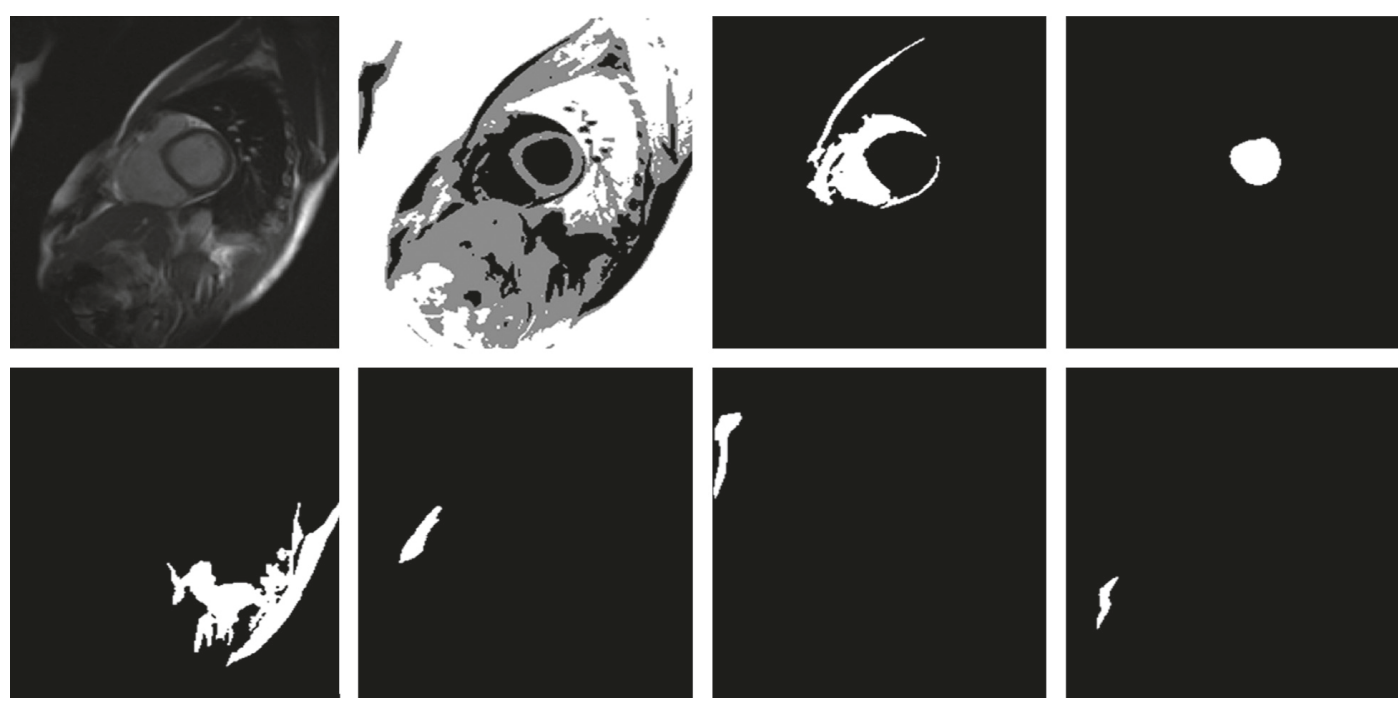

FIGURE 8: Sparse decomposition for endocardium segmentation of the left ventricle (the first is the original image, the second image shows the clustering result, and the rest are some basic functions with labeling for the clustering result).
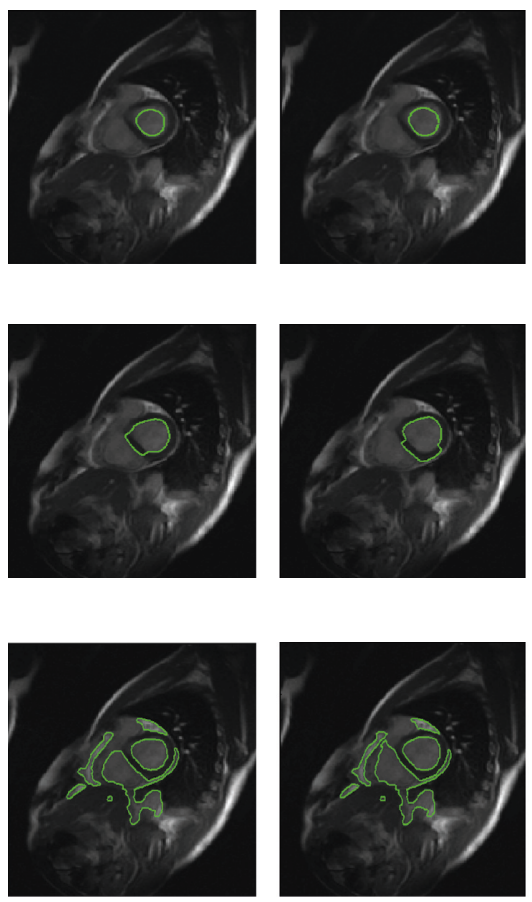
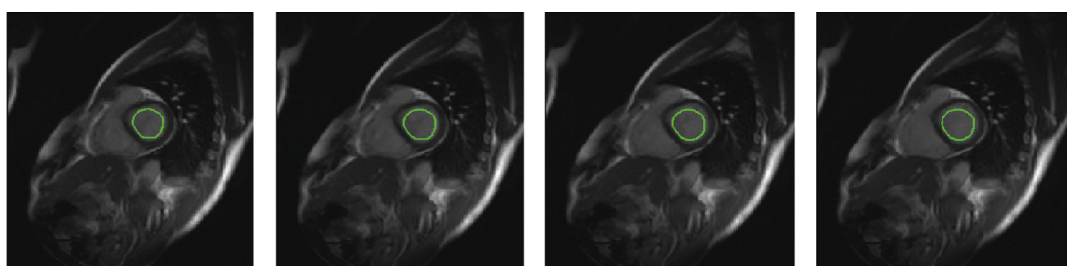

(a)
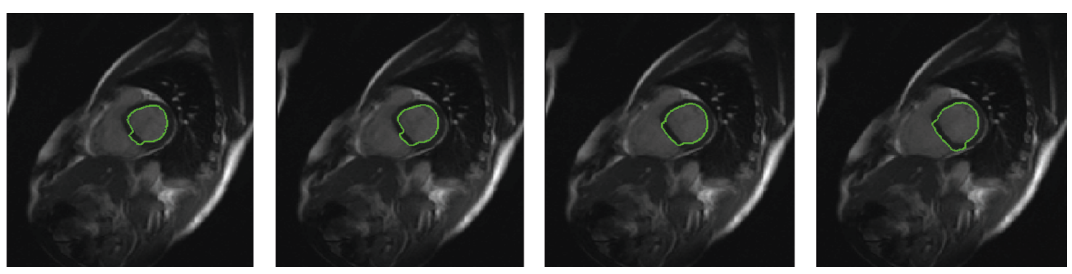

(b)
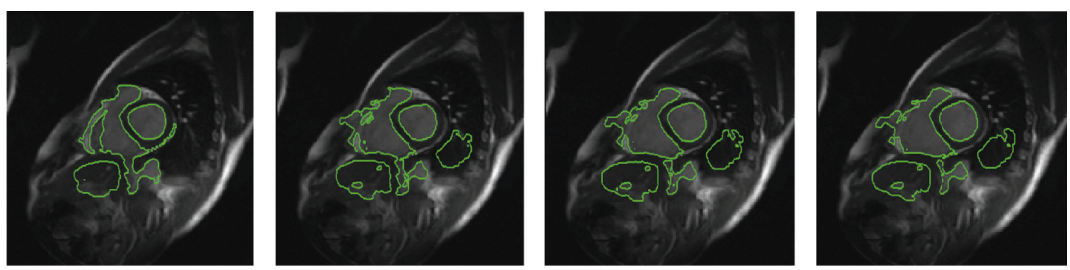

(c)

Figure 9: Continued. 

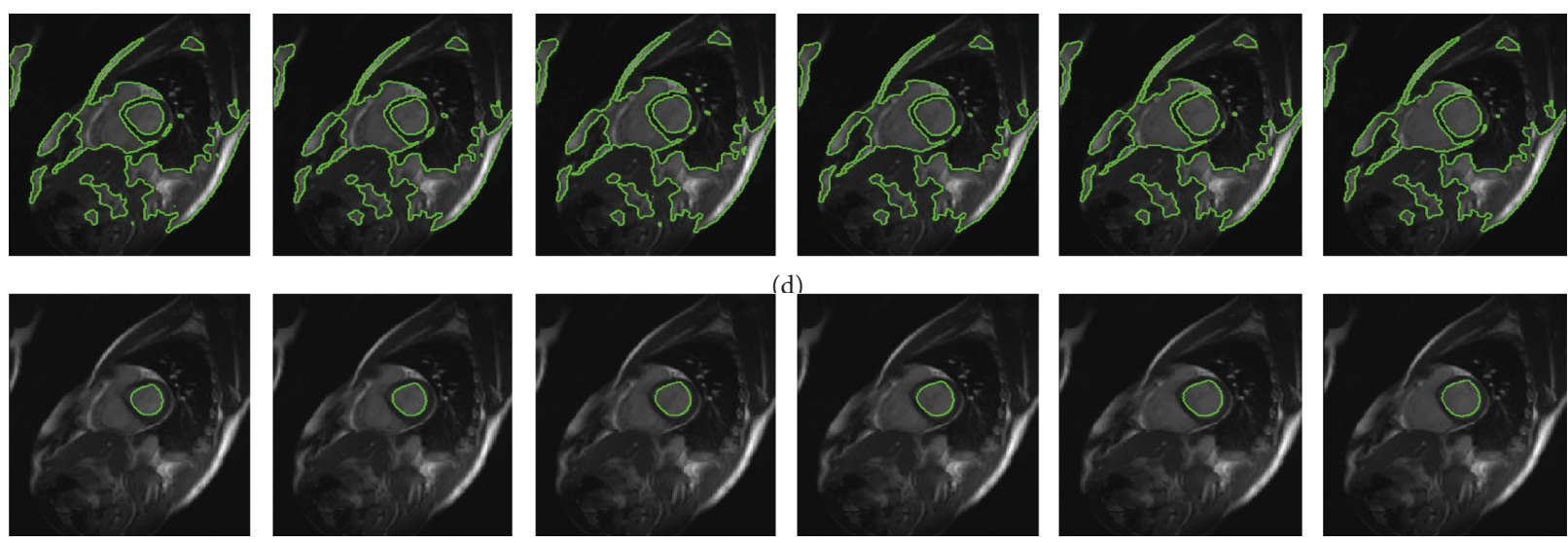

(e)

FIGURE 9: The endocardium segmentation results of the left ventricle in cardiac cine MRI. The segmentation results of (a) the ACGS model, (b) the DRLSE model, (c) the LSACM model, (d) the CFCV model, and (e) the proposed model with initialization of basic functions.

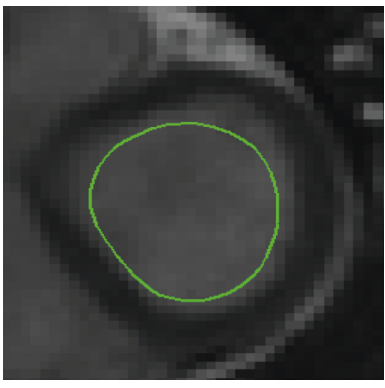

(a)

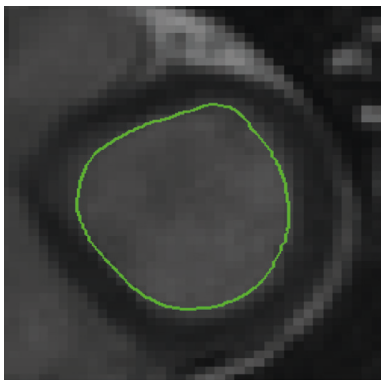

(b)

FIGURE 10: The zoomed comparison of ACGS and our method with the first column of Figure 9 (left: ACGS; right: our method).
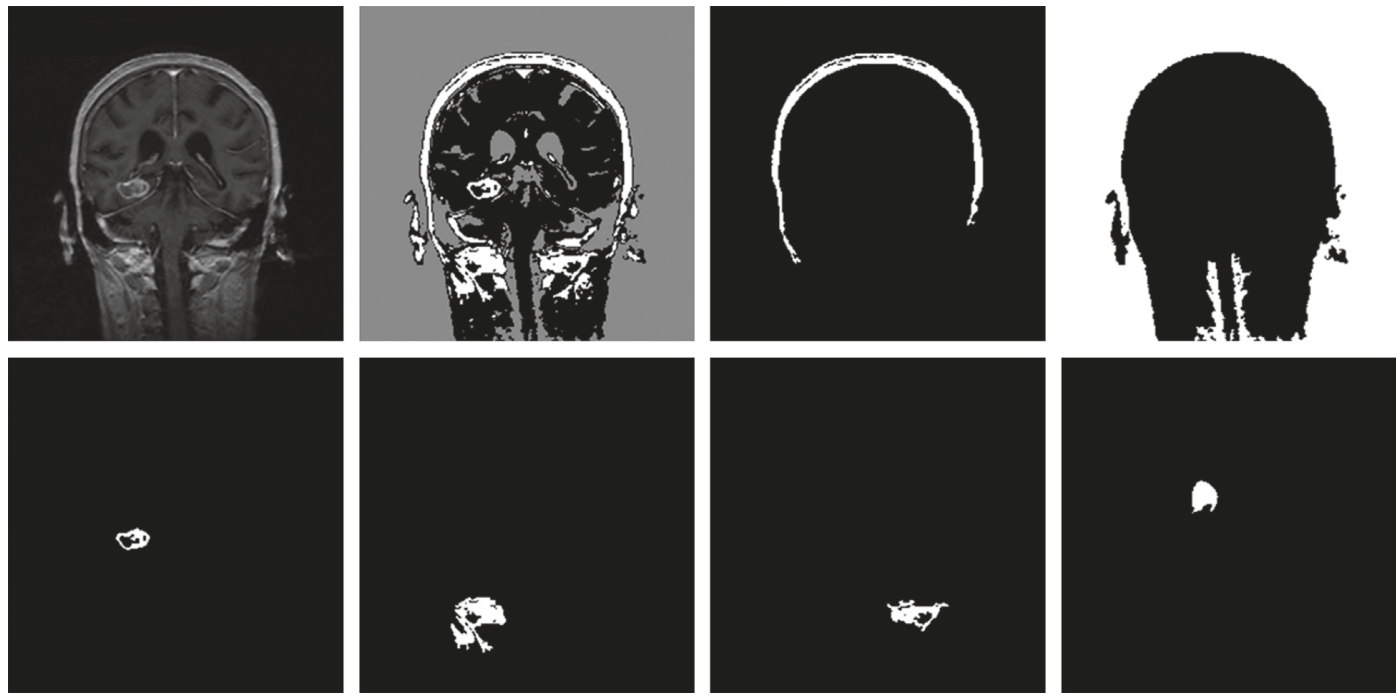

FIGURE 11: Sparse decomposition od brain MRI (the first is the original image, the second image shows the clustering result, and the rest are some basic functions with labeling for the clustering result.).

Compared with the right image of Figure 13, though the proposed method has obtained better results compared with other tested models, some boundaries of the tumor are not extracted in the left image. With regularized parameter $\lambda=15$, the extracted result is more accurate when the shape is less similar. 

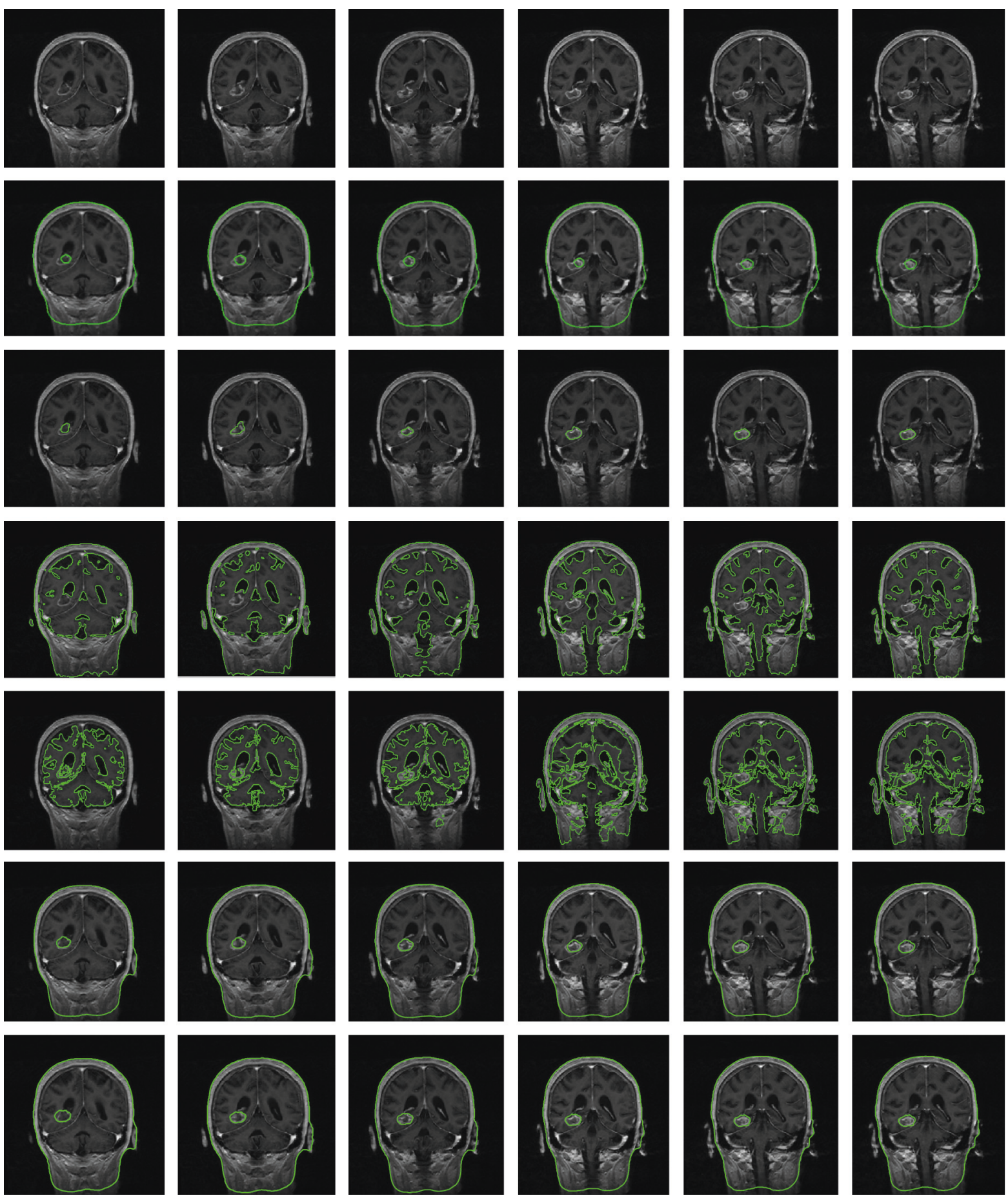

FIGURE 12: Segmentation results of brain tumor MRI (the rows from top to down: the original image, the results with ACGS, DRLSE, LSACM, and CFCV, the proposed method with default regularized parameter $\lambda=25$, and the proposed method with $\lambda=15$ ).

5.1. Quantitative Assessment. In order to objectively verify the accuracy of the simulation results of each model, this paper has carried out quantitative evaluation. That is to say, the segmentation results of the synthetic multitarget images are compared with the manual segmentation results. The metrics are the precision rate, recall rate, and F-Score calculated by F-Score algorithm.
It can be seen from Table 1 that the F-Score of the proposed model is usually higher than the other models. The reason is that although the LSACM and CFCV models can extract multiobject, these models always extract noise points and nontargets, and the accuracy of segmentation results is affected; The contour curve in the ACGS model cannot automatically split and merge, and the contour curve is 


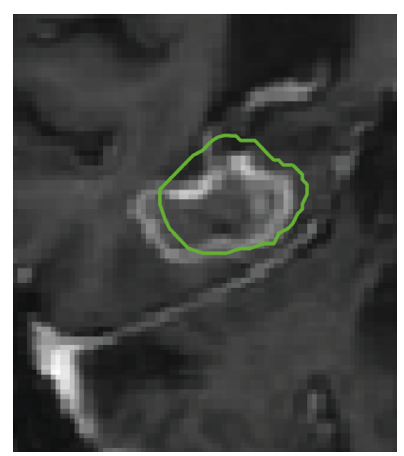

(a)

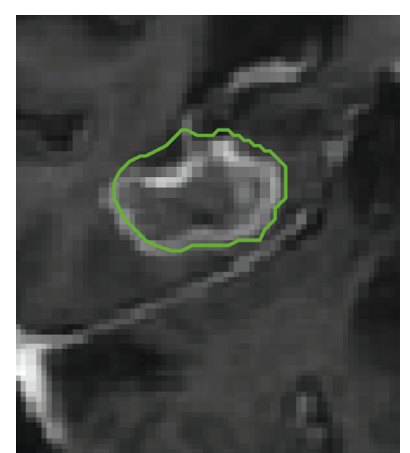

(b)

FIgURE 13: The zoomed comparisons for the fourth converged curves with our method in Figure 12 (left: $\lambda=25$, right $\lambda=15$ ).

entangled, especially, the two extracted objectives are relatively close to each other. The DRLSE model is robust to noise, but the effect of nontarget objects always decreases the segmentation accuracy. With proper and multiple initial contours, ACGS also extracts multiple objects. Compared with original ACGS, with the ideal initialization, the accuracy is improved. However, because of influence from other objects, the accuracy can be improved. The proposed model solves the problem that the ACGS model could not segment multiple targets with one initial contour, and the segmentation results are more accurate and more consistent with segmentation requirements.

According to the segmentation results shown in Figure 3, we use the precision rate, recall rate, and F-Score [41] calculated by the F-Score algorithm to objectively evaluate the segmentation performance of each model. The formula of the algorithm is defined as

$$
\left\{\begin{array}{l}
P=\frac{\mathrm{TP}}{\mathrm{TP}+\mathrm{FP}}, \\
R=\frac{\mathrm{TP}}{\mathrm{TP}+\mathrm{FN}}, \\
F=\frac{2 * \mathrm{PR}}{P+R},
\end{array}\right.
$$

where TP is the correct segmentation sample of the target foreground; FP is a segmentation sample that divides target background error into foreground, FN is a segmentation sample that divides the target foreground error into background, $P$ is the precision rate, which represents the proportion of the correct segmentation foreground to the total segmentation foreground, and its value is related to whether it is over segmentation, $R$ is the recall rate, which represents the proportion of the correct segmentation foreground to the standard target foreground sample, and its value is related to whether or not the leakage segmentation, and $F$ is the accuracy rate, which is the overall evaluation index used to determine the accuracy of segmentation results. The results obtained by the F-Score algorithm are shown in Table 1. In Tables 1 and 2, it is noted that ACGS(B) represents the ACGS model with basic functions initialization. Table 1 represents the accuracy of the segmentation results of the tested models.

From Table 1, we can see that compared to other models, the ACGS model, the CFCV model, LSACM model, and DRLSE model, the segmentation results of the proposed model are more ideal. The specific response is the precision rate, recall rate, and F-Score and there is no larger over segmentation or leakage segmentation, and the overall accuracy is higher than 95\%. The F-Score could show the overall evaluation index for each image, and the averaged F-Score for the tested images is also shown in Table 2. From Table 2, the average F-Score of the proposed method is observed to be the best. It is noted that the proposed method is not the best in each tested image. For the first image without feature missing, CFCV obtains the best segmentation accuracy. With ideal initialization, for the second tested example of Table 2, ACGS obtains the best segmentation result compared with other tested methods. However, the overall accuracy of the proposed method is the best in segmenting an image sequence, respectively, dealing with the images of sequence with feature missing.

\section{Conclusion}

In order to remedy the drawback that the ACGS model cannot segment the multitargets in sequence images with feature missing or misleading, an active contour model based on block similarity for multiple objects segmentation is proposed in this paper. For multitargets in one sequence of images, on the basis of sparse decomposition-based parametric active contour model, block similarity constraints are merged. In the parametric active contour model, the original shape features can be preserved when extracting multitarget objects. Finally, it is tested and applied in medical sequence of images. The robustness of the proposed model is verified.

\section{Data Availability}

The original data used to support the findings of this study can be obtained at http://www.cad.zju.edu.cn/home/xzhou/ code/acgs.zip. 


\section{Conflicts of Interest}

The authors declare that they have no conflicts of interest.

\section{Acknowledgments}

This work was jointly supported by the National Natural Science Foundation of China (Nos. U1404603, U1604154, and 61901160) and Natural Science Foundation of Henan Province (No. 162300410177).

\section{References}

[1] S. Zhu and A. Yuille, "Region competition: unifying snakes, region growing, and bayes/MDL for multiband image segmentation," IEEE Transactions on Pattern Analysis and Machine Intelligence, vol. 18, no. 9, pp. 884-900, 1996.

[2] C. Rother, V. Kolmogorov, and A. Blake, "GrabCut," ACM Transactions on Graphics, vol. 23, no. 3, pp. 309-314, 2004.

[3] J. Shen, Y. Du, and X. Li, "Interactive segmentation using constrained Laplacian optimization," IEEE Transactions on Circuits and Systems for Video Technology, vol. 24, no. 7, pp. 1088-1100, 2014.

[4] M. Kass, A. Witkin, and D. Terzopoulos, "Snakes: active contour models," International Journal of Computer Vision, vol. 1, no. 4, pp. 321-331, 1988.

[5] K. Zhang and H. Song, "Real-time visual tracking via online weighted multiple instance learning," Pattern Recognition, vol. 46, no. 1, pp. 397-411, 2013.

[6] Y. Wu, B. Ma, M. Yang, Y. Jia, and J. Zhang, "Metric learning based structural appearance model for robust visual tracking," IEEE Transactions on Circuits and Systems for Video Technology, vol. 24, no. 5, pp. 865-877, 2014.

[7] L. D. Cohen and I. Cohen, "Finite-element methods for active contour models and balloons for 2-D and 3-D images," IEEE Transactions on Pattern Analysis and Machine Intelligence, vol. 15, no. 11, pp. 1131-1147, November, 1993.

[8] S. Osher and J. A. Sethian, "Fronts propagating with curvature-dependent speed: algorithms based on Hamilton-Jacobi formulations," Journal of Computational Physics, vol. 79, no. 1, pp. 12-49, 1988.

[9] V. Caselles, R. Kimmel, and G. Sapiro, "Geodesic active contours," International Conference on Computer Vision, vol. 22, no. 1, pp. 694-699, 1995.

[10] K. Zhang, H. Song, and L. Zhang, "Active contours driven by local image fitting energy," Pattern Recognition, vol. 43, no. 4, pp. 1199-1206, 2010.

[11] X. Han, C. Xu, and J. L. Prince, “A topology preserving level set method for geometric deformable models," IEEE Transactions on Pattern Analysis and Machine Intelligence, vol. 25, no. 6, pp. 755-768, 2003.

[12] L. Wang, G. Chen, D. Shi et al., "Active contours driven by edge entropy fitting energy for image segmentation," Signal Processing, vol. 149, pp. 27-35, 2018.

[13] C. M. Li, C. Kao, J. Gore, and Z. Ding, "Implicit active contours driven by local binary fitting energy," in Proceedings of the IEEE Conference on Computer Vision and Pattern Recognition (CVPR), pp. 1-7, Anchorage, Alaska, November 2007.

[14] F. Dong, Z. Chen, and J. Wang, "A new level set method for inhomogeneous image segmentation," Image and Vision Computing, vol. 31, no. 10, pp. 809-822, 2013.

[15] X. Zhou, X. Huang, J. S. Duncan et al., "Active contours with group similarity," in Proceedings of the IEEE Conference on
Computer Vision and Pattern Recognition (CVPR), pp. 2969-2976, Columbus, OH, USA, June 2013.

[16] C. Li, C. Xu, C. Gui, and M. D. Fox, "Distance regularized level set evolution and its application to image segmentation," IEEE Transactions on Image Processing, vol. 19, no. 12, pp. 32433254, 2010.

[17] T. F. Chan and L. A. Vese, "Active contours without edges," IEEE Transactions on Image Processing, vol. 10, no. 2, pp. 266-277, 2001.

[18] E. S. Brown, T. F. Chan, and X. Bresson, "Completely convex formulation of the Chan-Vese image segmentation model," International Journal of Computer Vision, vol. 98, no. 1, pp. 103-121, 2012.

[19] K. Zhang, L. Zhang, K.-M. Lam, and D. Zhang, "A level set approach to image segmentation with intensity inhomogeneity," IEEE Transactions on Cybernetics, vol. 46, no. 2, pp. 546-557, 2016.

[20] W. Tao, K. Li, and K. Sun, "SaCoseg: object cosegmentation by shape conformability," IEEE Transactions on Image Processing, vol. 24, no. 3, pp. 943-955, 2015.

[21] N. Paragios, "A level set approach for shape-driven segmentation and tracking of the left ventricle," IEEE Transactions on Medical Imaging, vol. 22, no. 6, pp. 773-776, 2003.

[22] S. Zhang, Y. Zhan, M. Dewan, J. Huang, D. N. Metaxas, and X. S. Zhou, "Towards robust and effective shape modeling: sparse shape composition," Medical Image Analysis, vol. 16, no. 1, pp. 265-277, 2012.

[23] D. Cremers, "Dynamical statistical shape priors for level setbased tracking," IEEE Transactions on Pattern Analysis and Machine Intelligence, vol. 28, no. 8, pp. 1262-1273, 2006.

[24] F. Chen, H. Yu, R. Hu et al., "Deep learning shape priors for object segmentation," in Proceedings of the IEEE Conference on Computer Vision and Pattern Recognition (CVPR), pp. 1870-1877, Columbus, OH, USA, June 2013.

[25] T.-T. Tran, V.-T. Pham, and K.-K. Shyu, "Moment-based alignment for shape prior with variational B-spline level set," Machine Vision and Applications, vol. 24, no. 5, pp. 10751091, 2013.

[26] N. Ben-Zadok, T. Riklin-Raviv, and N. Kiryati, "Interactive level set segmentation for image-guided therapy," in Proceedings of the IEEE International Conference on Symposium on Biomedical Imaging: From Nano To Macro, pp. 1079-1082, Boston, MA, USA, June 2009.

[27] N. Ben-Zadok, T. Riklin-Raviv, and N. Kiryati, Interactive Level Set Segmentation for Image-Guided Therapy: Examples, Institute of Electrical and Electronics Engineers, Piscataway, NJ, USA, 2009, http://www.eng.tau.ac.il/nk/ISBI09/.

[28] S. Zhang, Y. Zhan, and D. N. Metaxas, "Deformable segmentation via sparse representation and dictionary learning," Medical Image Analysis, vol. 16, no. 7, pp. 1385-1396, 2012.

[29] K. Zhang, L. Zhang, H. Song, and D. Zhang, "Reinitializationfree level set evolution via reaction diffusion," IEEE Transactions on Image Processing, vol. 22, no. 1, pp. 258-271, January, 2013.

[30] S. Zhang, Y. Zhan, M. Dewan, J. Huang, D. N. Metaxas, and X. S. Zhou, "Towards robust and effective shape modeling: sparse shape composition," Medical Image Analysis, vol. 16, no. 1, pp. 265-277, 2012.

[31] M. Zhou, H. Chen, J. Paisley et al., "Non-parametric Bayesian dictionary learning for sparse image representations," in Proceedings of the International Conference on Neural Information Processing Systems, pp. 2295-2303, Vancouver, Canada, December 2009. 
[32] A. K. Mishra and P. W. Fieguth, Partial Differential Equations and the Calculus of Variations, Springer, New York, NY, USA, 2006.

[33] C. Xu and J. L. Prince, "Snakes, shapes, and gradient vector flow," IEEE Transactions on Image Processing, vol. 7, no. 3, pp. 359-369, 1998.

[34] B. Li and S. T. Acton, "Active contour external force using vector field convolution for image segmentation," IEEE Transactions on Image Processing, vol. 16, no. 8, pp. 20962106, 2007.

[35] Q. Li, T. Deng, and W. Xie, "Active contours driven by divergence of gradient vector flow," Signal Processing, vol. 120, pp. 185-199, 2016.

[36] L. Qin, C. Zhu, Y. Zhao, H. Bai, and H. Tian, "Generalized gradient vector flow for snakes: new observations, analysis, and improvement," IEEE Transactions on Circuits and Systems for Video Technology, vol. 23, no. 5, pp. 883-897, 2013.

[37] G. Liu and M. Deng, "Parametric active contour based on sparse decomposition for multi-objects extraction," Signal Processing, vol. 148, pp. 314-321, 2018.

[38] J. A. Tropp and A. C. Gilbert, "Signal recovery from random measurements via orthogonal matching pursuit," IEEE Transactions on Information Theory, vol. 53, no. 12, pp. 4655-4666, 2007.

[39] G. Liu, H. Li, and L. Yang, "A topology preserving method of evolving contours based on sparsity constraint for object segmentation," IEEE Access, vol. 5, pp. 19971-19982, 2017.

[40] J. C. Bezdek, Pattern Recognition with Fuzzy Objective Function Algorithms, Plenum Press, New York, NY, USA, 1981.

[41] A. Kovacs and T. Sziranyi, "Harris function based active contour external force for image segmentation," Pattern Recognition Letters, vol. 33, no. 9, pp. 1180-1187, 2012. 


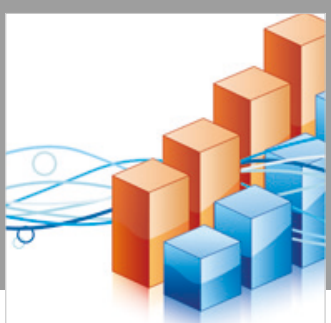

Advances in

Operations Research

\section{-n-m}
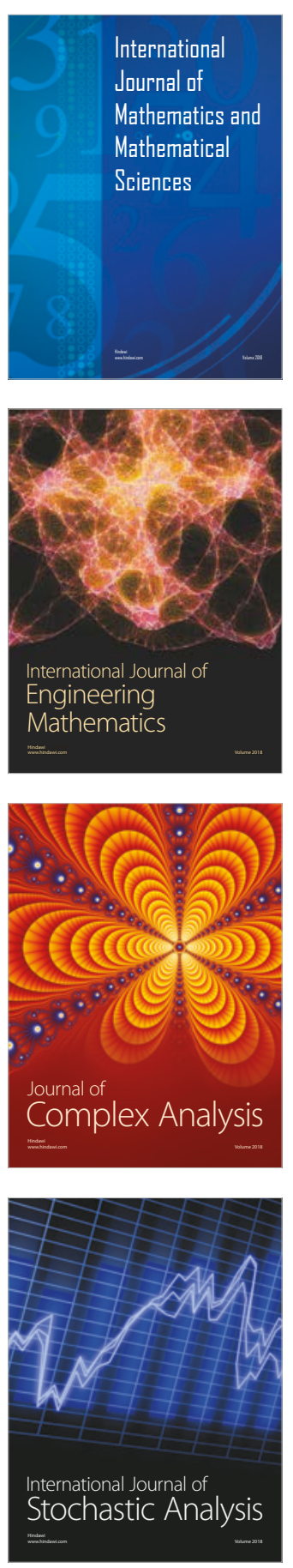
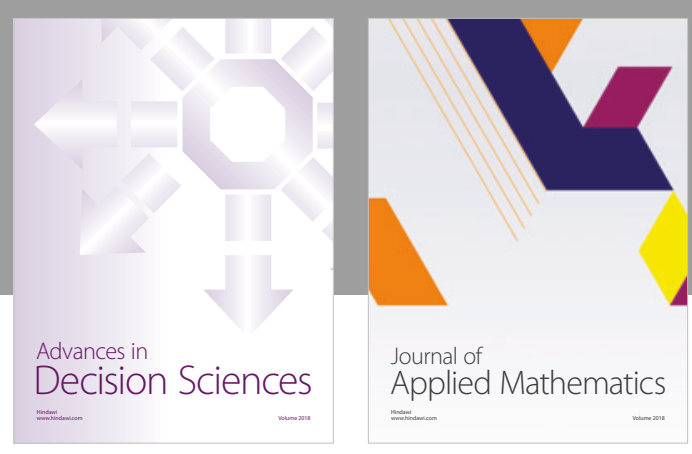

Journal of

Applied Mathematics
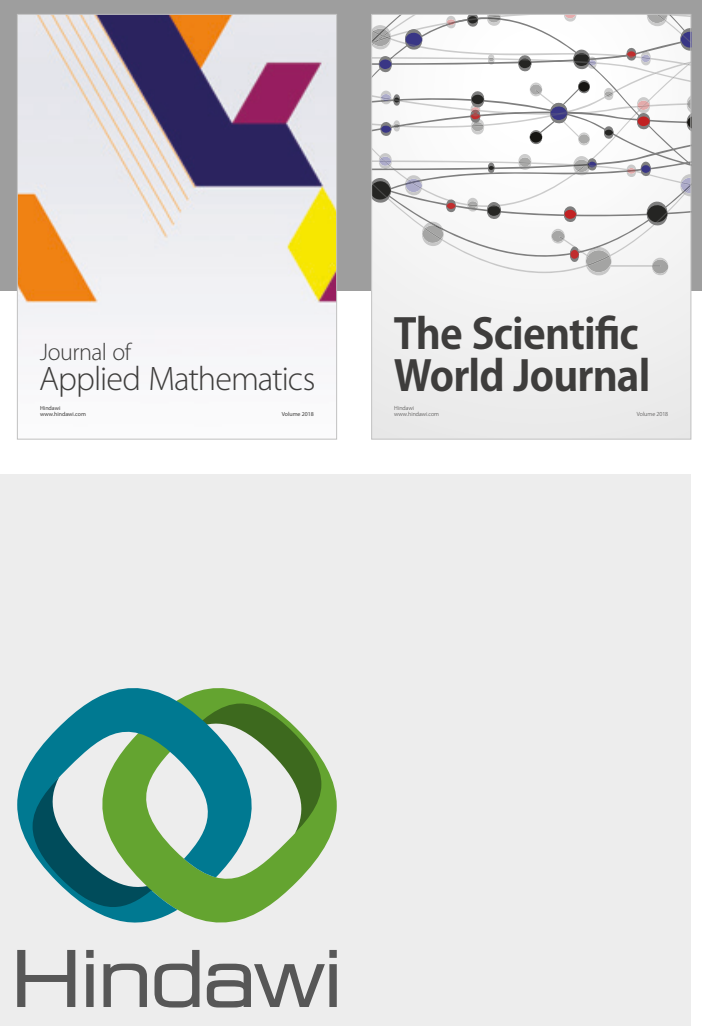

Submit your manuscripts at

www.hindawi.com

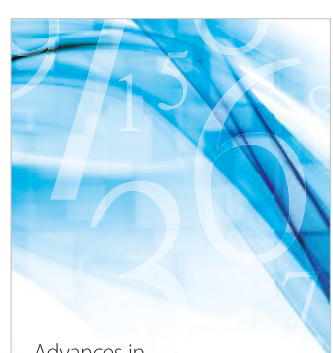

Advances in
Numerical Analysis
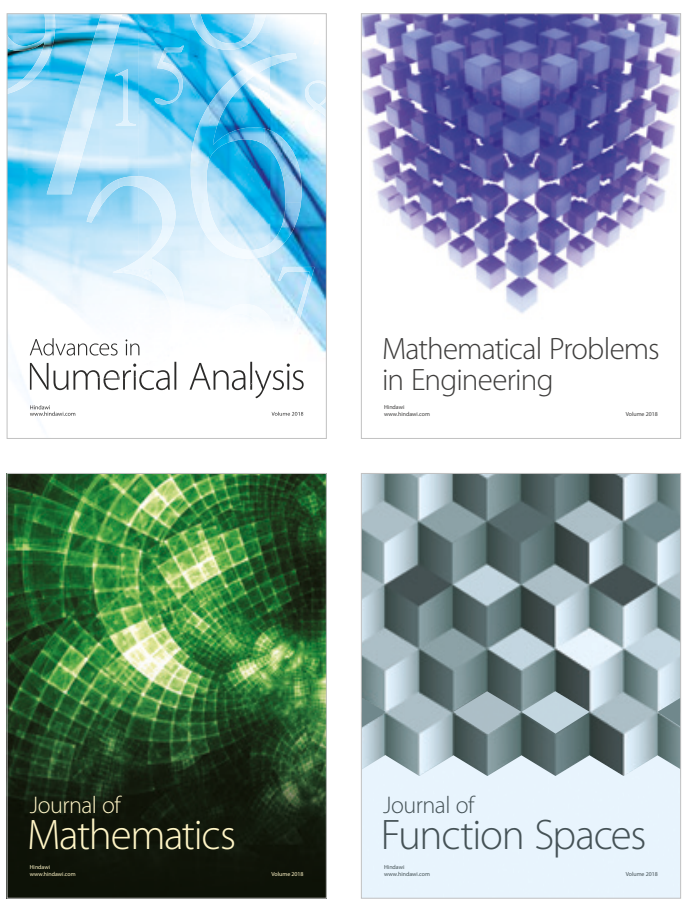

Mathematical Problems in Engineering

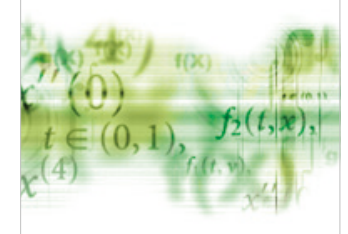

International Journal of

Differential Equations

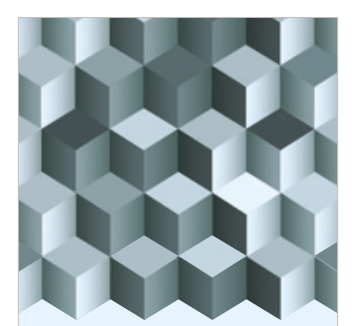

Journal of

Function Spaces
The Scientific

World Journal

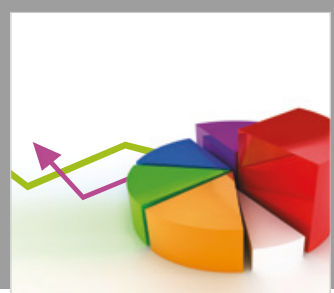

Journal of

Probability and Statistics
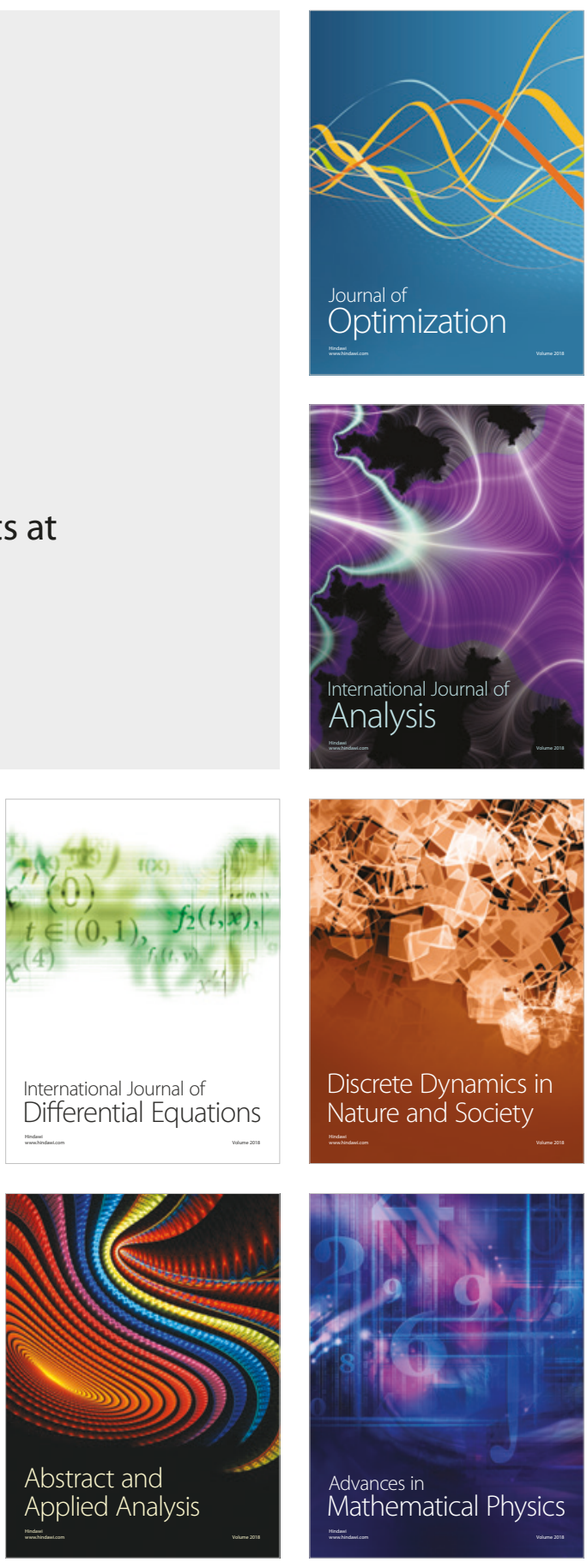